\title{
Social Network Games
}

\author{
Sunil Simon \\ Centre for Mathematics and Computer Science (CWI), Amsterdam \\ E-mail: s.e.simon@cwi.nl \\ Krzysztof R. Apt
Centre for Mathematics and Computer Science (CWI) and ILLC, University of Amsterdam, The Netherlands
E-mail: k.r.apt@cwi.nl

\begin{abstract}
One of the natural objectives of the field of the social networks is to predict agents' behaviour. To better understand the spread of various products through a social network [2] introduced a threshold model, in which the nodes influenced by their neighbours can adopt one out of several alternatives. To analyze the consequences of such product adoption we associate here with each such social network a natural strategic game between the agents.

In these games the payoff of each player weakly increases when more players choose his strategy, which is exactly opposite to the congestion games. The possibility of not choosing any product results in two special types of (pure) Nash equilibria.

We show that such games may have no Nash equilibrium and that determining an existence of a Nash equilibrium, also of a special type, is NP-complete. This implies the same result for a more general class of games, namely polymatrix games. The situation changes when the underlying graph of the social network is a DAG, a simple cycle, or, more generally, has no source nodes. For these three classes we determine the complexity of an existence of (a special type of) Nash equilibria.

We also clarify for these categories of games the status and the complexity of the finite best response property (FBRP) and the finite improvement property (FIP). Further, we introduce a new property of the uniform FIP which is satisfied when the underlying graph is a simple cycle, but determining it is co-NP-hard in the general case and also when the underlying graph has no source nodes. The latter complexity results also hold for the property of being a weakly acyclic game. A preliminary version of this paper appeared as [19].
\end{abstract}

Keywords: Social networks, strategic games, Nash equilibrium, finite improvement property, complexity. 


\section{Introduction}

\subsection{Background}

Social networks are a thriving interdisciplinary research area with links to sociology, economics, epidemiology, computer science, and mathematics. A flurry of numerous articles and recent books, see, e.g., [8], testifies to the relevance of this field. It deals with such diverse topics as epidemics, analysis of the connectivity, spread of certain patterns of social behaviour, effects of advertising, and emergence of 'bubbles' in financial markets.

One of the prevalent types of models of social networks are the threshold models introduced in [9] and [18]. In such a setup each node $i$ has a threshold $\theta(i) \in(0,1]$ and adopts an 'item' given in advance (which can be a disease, trend, or a specific product) when the total weight of incoming edges from the nodes that have already adopted this item exceeds the threshold. One of the most important issues studied in the threshold models has been that of the spread of an item, see, e.g., [17, 14, 7]. From now on we shall refer to an 'item' that is spread by a more specific name of a 'product'.

In this context very few papers dealt with more than one product. One of them is [11] with its focus on the notions of compatibility and bilinguality that result when one adopts both available products at an extra cost. Another one is [5], where the authors investigate whether the algorithmic approach of [14] can be extended to the case of two products.

In [2] we introduced a new threshold model of a social network in which nodes (agents) influenced by their neighbours can adopt one out of several products. This model allowed us to study various aspects of the spread of a given product through a social network, in the presence of other products. We analysed from the complexity point of view the problems of determining whether adoption of a given product by the whole network is possible (respectively, necessary), and when a unique outcome of the adoption process is guaranteed. We also clarified for social networks without unique outcomes the complexity of determining whether a given node has to adopt some (respectively, a given) product in some (respectively, all) final network(s), and the complexity of computing the minimum and the maximum possible spread of a given product.

\subsection{Motivation}

We are interested in understanding and predicting the behaviour of the consumers (agents) who form a social network and are confronted with several alternatives (products). To carry out such an analysis we use the above model of [2] and associate with each such social network a natural strategic game. In this game the strategies of an agent are products he can choose. Additionally a 'null' strategy is available that models the decision of not choosing any product. The idea is that after each agent chose a product, or decided not to choose any, the agents assess the optimality of their choices comparing them to the choices made by their neighbours. This leads to a natural study of (pure) Nash equilib- 
ria, in particular of those in which some, respectively all, constituent strategies are non-null.

Social network games are related to graphical games of [13], in which the payoff function of each player depends only on a (usually small) number of other players. In this work the focus was mainly on finding mixed (approximate) Nash equilibria. However, in graphical games the underlying structures are undirected graphs. Also, social network games exhibit the following join the crowd property:

the payoff of each player weakly increases when more players choose his strategy.

(We define this property more precisely in Subsection 2.3.)

Since these games are related to social networks, some natural special cases are of interest: when the underlying graph is a DAG or has no source nodes, with the special case of a simple cycle. Such social networks correspond respectively to a hierarchical organization or to a 'circle of friends', in which everybody has a friend (a neighbour). Studying Nash equilibria of these games and various properties defined in terms of improvement paths allows us to gain better insights into the consequences of adopting products.

\subsection{Related work}

There are a number of papers that focus on games associated with various forms of networks, see, e.g., [20] for an overview. A more recent example is [1] that analyses a strategic game between players being firms who select nodes in an undirected graph in order to advertise competing products via 'viral marketing'. However, in spite of the focus on similar questions concerning the existence and structure of Nash equilibria and on their reachability, from a technical point of view, the games studied here seem to be unrelated to the games studied elsewhere.

Still, it is useful to mention the following phenomenon. When the underlying graph of a social network has no source nodes, the game always has a trivial Nash equilibrium in which no agent chooses a product. A similar phenomenon has been recently observed in [6] in the case of their network formation games, where such equilibria are called degenerate. Further, note that the 'join the crowd' property is exactly the opposite of the defining property of the congestion games with player-specific payoff functions introduced in [15]. In these game the payoff

of each player weakly decreases when more players choose his strategy. Because in our case (in contrast to [15]) the players can have different strategy sets, the resulting games are not coordination games.

\subsection{Plan of the paper and summary of the results}

In the next section we recall the model of social networks introduced in [2] and define strategic games associated with these networks. Next, in Section 3 we 
show that in general Nash equilibria do not need to exist even if we limit ourselves to a special class of networks in which for each node all its neighbours have the same weight. We prove that determining an existence of a Nash equilibrium is NP-complete, also when we limit our attention to the two special types of equilibria. Then in Section 4 we show that this NP-completeness result holds for a more general class of games, namely polymatrix games.

Motivated by these results we consider in Section 5 strategic games associated with three classes of social networks, the ones whose underlying graph is a DAG, a simple cycle, or, more generally, has no source nodes. For each class we determine the complexity of deciding whether a Nash equilibrium (possibly of a special type) exists. We also show that for these games the price of anarchy and the price of stability are unbounded.

Next, in Section 6 we study the finite best response property (FBRP) of [16]. We prove that deciding whether a game associated with a social network has the FBRP is co-NP-hard. Then, in Section 7, we consider the above three classes of games. We show that when the underlying graph is a DAG or there are just two nodes, the game has the FBRP, that there is an efficient algorithm when the underlying graph is a simple cycle, and that the problem is co-NPhard when the underlying graph has no source nodes. In Sections 8 and 9 we obtain analogous results for the finite improvement property (FIP), though the complexity when the underlying graph is a simple cycle remains open.

In Section 10 we introduce a new property, that we call the uniform FIP, that is of independent interest. We show that deciding whether a game associated with a social network has the uniform FIP is co-NP-hard. Then, we study in Section 11 the special cases. We show that when the underlying graph is a simple cycle the game has the uniform FIP. However, when the underlying graph has no source nodes the problem is co-NP-hard. In Section 12 we show that the property of having the uniform FIP is stronger than that of being weakly acyclic (see [21] and [15]). Determining whether a game is weakly acyclic is also co-NP-hard, also when the underlying graph has no source nodes

Finally, in Section 13 we summarize the obtained complexity results and suggest some further research.

\section{Preliminaries}

\section{$2.1 \quad$ Strategic games}

Assume a set $\{1, \ldots, n\}$ of players, where $n>1$. A strategic game for $n$ players, written as $\left(S_{1}, \ldots, S_{n}, p_{1}, \ldots, p_{n}\right)$, consists of a non-empty set $S_{i}$ of strategies and a payoff function $p_{i}: S_{1} \times \ldots \times S_{n} \rightarrow \mathbb{R}$, for each player $i$.

Fix a strategic game $G:=\left(S_{1}, \ldots, S_{n}, p_{1}, \ldots, p_{n}\right)$. We denote $S_{1} \times \cdots \times S_{n}$ by $S$, call each element $s \in S$ a joint strategy, denote the $i$ th element of $s$ by $s_{i}$, and abbreviate the sequence $\left(s_{j}\right)_{j \neq i}$ to $s_{-i}$. Occasionally we write $\left(s_{i}, s_{-i}\right)$ instead of $s$.

We call a strategy $s_{i}$ of player $i$ a best response to a joint strategy $s_{-i}$ of 
his opponents if $\forall s_{i}^{\prime} \in S_{i} p_{i}\left(s_{i}, s_{-i}\right) \geq p_{i}\left(s_{i}^{\prime}, s_{-i}\right)$. We call a joint strategy $s$ a

Nash equilibrium if each $s_{i}$ is a best response to $s_{-i}$, that is, if

$$
\forall i \in\{1, \ldots, n\} \forall s_{i}^{\prime} \in S_{i} p_{i}\left(s_{i}, s_{-i}\right) \geq p_{i}\left(s_{i}^{\prime}, s_{-i}\right)
$$

Further, we call a strategy $s_{i}^{\prime}$ of player $i$ a better response given a joint strategy $s$ if $p_{i}\left(s_{i}^{\prime}, s_{-i}\right)>p_{i}\left(s_{i}, s_{-i}\right)$.

Given a joint strategy $s$ we call the $\operatorname{sum} S W(s)=\sum_{j=1}^{n} p_{j}(s)$ the social welfare of $s$. When the social welfare of $s$ is maximal we call $s$ a social optimum. Recall that, given a finite game that has a Nash equilibrium, its price of anarchy (respectively, price of stability) is the ratio $\frac{S W(s)}{S W\left(s^{\prime}\right)}$ where $s$ is a social optimum and $s^{\prime}$ is a Nash equilibrium with the lowest (respectively, highest) social welfare. In the case of division by zero, we interpret the outcome as $\infty$.

Following the terminology of [16], a path in $S$ is a sequence $\left(s^{1}, s^{2}, \ldots\right)$ of joint strategies such that for every $k>1$ there is a player $i$ such that $s^{k}=$ $\left(s_{i}^{\prime}, s_{-i}^{k-1}\right)$ for some $s_{i}^{\prime} \neq s_{i}^{k-1}$. A path is called an improvement path if it is maximal and for all $k>1, p_{i}\left(s^{k}\right)>p_{i}\left(s^{k-1}\right)$ where $i$ is the player who deviated from $s^{k-1}$. If an improvement path satisfies the additional property that $s_{i}^{k}$ is a best response to $s_{-i}^{k-1}$ for all $k>1$ then it is called a best response improvement path.

The last condition simply means that each deviating player selects a better (best) response. A game has the finite improvement property (FIP), (respectively, the finite best response property $(\boldsymbol{F B R P}))$ if every improvement path (respectively, every best response improvement path) is finite. Obviously, if a game has the FIP or the FBRP, then it has a Nash equilibrium - it is the last element of each path. Finally, a game is called weakly acyclic (see $[21,15])$ if for every joint strategy there exists a finite improvement path that starts at it.

\subsection{Social networks}

We are interested in specific strategic games defined over social networks. In what follows we focus on a model of the social networks recently introduced in $[2]$.

Let $V=\{1, \ldots, n\}$ be a finite set of agents and $G=(V, E, w)$ a weighted directed graph with $w_{i j} \in[0,1]$ being the weight of the edge $(i, j)$. We assume that $G$ does not have self loops, i.e., for all $i \in\{1, \ldots, n\},(i, i) \notin E$. We often use the notation $i \rightarrow j$ to denote $(i, j) \in E$ and write $i \rightarrow^{*} j$ if there is a path from $i$ to $j$ in the graph $G$. Given a node $i$ of $G$ we denote by $N(i)$ the set of nodes from which there is an incoming edge to $i$. We call each $j \in N(i)$ a neighbour of $i$ in $G$. We assume that for each node $i$ such that $N(i) \neq \emptyset$, $\sum_{j \in N(i)} w_{j i} \leq 1$. An agent $i \in V$ is said to be a source node in $G$ if $N(i)=\emptyset$.

Let $\mathcal{P}$ be a finite set of alternatives or products. By a social network (from now on, just network) we mean a tuple $\mathcal{S}=(G, \mathcal{P}, P, \theta)$, where $P$ assigns to each agent $i$ a non-empty set of products $P(i)$ from which it can make a choice. 
$\theta$ is a threshold function that for each $i \in V$ and $t \in P(i)$ yields a value $\theta(i, t) \in(0,1]$.

Given a network $\mathcal{S}$ we denote by source $(\mathcal{S})$ the set of source nodes in the underlying graph $G$. One of the classes of the networks we shall study are the ones with $\operatorname{source}(\mathcal{S})=\emptyset$. We call a network equitable if the weights are defined as $w_{j i}=\frac{1}{|N(i)|}$ for all nodes $i$ and $j \in N(i)$.

\subsection{Social network games}

Fix a network $\mathcal{S}=(G, \mathcal{P}, P, \theta)$. Each agent can adopt a product from his product set or choose not to adopt any product. We denote the latter choice by $t_{0}$.

With each network $\mathcal{S}$ we associate a strategic game $\mathcal{G}(\mathcal{S})$. The idea is that the nodes simultaneously choose a product or abstain from choosing any. Subsequently each node assesses his choice by comparing it with the choices made by his neighbours. Formally, we define the game as follows:

- the players are the agents,

- the set of strategies for player $i$ is $S_{i}:=P(i) \cup\left\{t_{0}\right\}$,

- for $i \in V, t \in P(i)$ and a joint strategy $s$, let $\mathcal{N}_{i}^{t}(s):=\left\{j \in N(i) \mid s_{j}=t\right\}$, i.e., $\mathcal{N}_{i}^{t}(s)$ is the set of neighbours of $i$ who adopted the product $t$ in $s$.

The payoff function is defined as follows, where $c_{0}$ is some positive constant given in advance:

- for $i \in \operatorname{source}(\mathcal{S})$,

$$
p_{i}(s):= \begin{cases}0 & \text { if } s_{i}=t_{0} \\ c_{0} & \text { if } s_{i} \in P(i)\end{cases}
$$

- for $i \notin \operatorname{source}(\mathcal{S})$,

$$
p_{i}(s):= \begin{cases}0 & \text { if } s_{i}=t_{0} \\ \left(\sum_{j \in \mathcal{N}_{i}^{t}(s)} w_{j i}\right)-\theta(i, t) & \text { if } s_{i}=t, \text { for some } t \in P(i)\end{cases}
$$

Let us explain the underlying motivations behind the above definition. In the first item we assume that the payoff function for the source nodes is constant only for simplicity. In the last section of the paper we explain that the obtained results hold equally well in the case when the source nodes have arbitrary positive utility for each product.

The second item in the payoff definition is motivated by the following considerations. When agent $i$ is not a source node, his 'satisfaction' from a joint strategy depends positively on the accumulated weight (read: 'influence') of his neighbours who made the same choice as he did, and negatively from his threshold level (read: 'resistance') to adopt this product. The assumption that $\theta(i, t)>0$ reflects the view that there is always some resistance to adopt a product. So when this resistance is high, it can happen that the payoff is negative. 
Of course, in such a situation not adopting any product, represented by the strategy $t_{0}$, is a better alternative.

The presence of this possibility allows each agent to refrain from choosing a product. This refers to natural situations, such as deciding not to purchase a smartphone or not going on vacation. In the last section we refer to an initiated research on social network games in which the strategy $t_{0}$ is not present. Such games capture situations in which the agents have to take some decision, for instance selecting a secondary school for their children.

By definition the payoff of each player depends only on the strategies chosen by his neighbours, so social network games are related to graphical games of [13]. However, the underlying dependence structure of a social network game is a directed graph and the presence of the special strategy $t_{0}$ available to each player makes these games more specific. Also, as already mentioned in Subsection 1.2, these games satisfy the join the crowd property that we define as follows:

Each payoff function $p_{i}$ depends only on the strategy chosen by player $i$ and the set of players who also chose his strategy. Moreover, the dependence on this set is monotonic.

In what follows for $t \in \mathcal{P} \cup\left\{t_{0}\right\}$ we use the notation $\bar{t}$ to denote the joint strategy $s$ where $s_{j}=t$ for all $j \in V$. This notation is legal only if for all agents $i$ it holds that $t \in P(i)$.

The presence of the strategy $t_{0}$ motivates the introduction and study of special types of Nash equilibria. We say that a Nash equilibrium $s$ is

- determined if for all $i, s_{i} \neq t_{0}$,

- non-trivial if for some $i, s_{i} \neq t_{0}$,

- trivial if for all $i, s_{i}=t_{0}$, i.e., $s=\overline{t_{0}}$.

\section{Nash equilibria: general case}

The first natural question that we address is that of the existence of Nash equilibria in social network games. We establish the following result.

Theorem 1. Deciding whether for a network $\mathcal{S}$ the game $\mathcal{G}(\mathcal{S})$ has a (respectively, non-trivial) Nash equilibrium is NP-complete.

To prove it we first construct an example of a social network game with no Nash equilibrium and then use it to determine the complexity of the existence of Nash equilibria.

Example 2. Consider the network given in Figure 1, where the product set of each agent is marked next to the node denoting it and the weights are labels on the edges. The source nodes are represented by the unique product in the product set. 


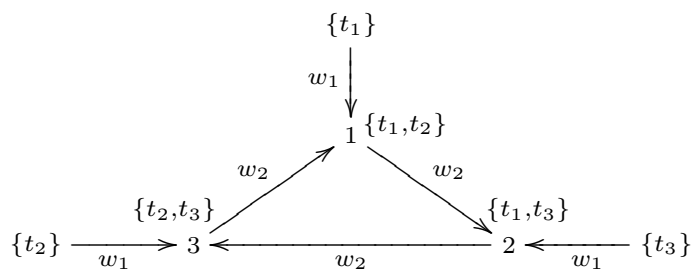

Figure 1: A network with no Nash equilibrium

So the weights on the edges from the nodes $\left\{t_{1}\right\},\left\{t_{2}\right\},\left\{t_{3}\right\}$ are marked by $w_{1}$ and the weights on the edges forming the triangle are marked by $w_{2}$. We assume that each threshold is a constant $\theta$, where $\theta<w_{1}<w_{2}$. So it is more profitable to a player residing on the triangle to adopt the product adopted by his neighbour residing on a triangle than by the other neighbour who is a source node. For convenience we represent each joint strategy as a triple of strategies of players 1,2 and 3 .

It is easy to check that in the game associated with this network no joint strategy is a Nash equilibrium. Indeed, each agent residing on the triangle can secure a payoff of at least $w_{1}-\theta>0$, so it suffices to analyze the joint strategies in which $t_{0}$ is not used. There are in total eight such joint strategies. Here is their listing, where in each joint strategy we underline the strategy that is not a best response to the choice of other players: $\left(\underline{t_{1}}, t_{1}, t_{2}\right),\left(t_{1}, t_{1}, \underline{t_{3}}\right),\left(t_{1}, t_{3}, \underline{t_{2}}\right)$, $\left(t_{1}, \underline{t_{3}}, t_{3}\right),\left(t_{2}, \underline{t_{1}}, t_{2}\right),\left(t_{2}, \underline{t_{1}}, t_{3}\right),\left(t_{2}, t_{3}, \underline{t_{2}}\right),\left(\underline{t_{2}}, t_{3}, t_{3}\right)$.

\section{Proof of Theorem 1.}

As in [2] we use a reduction from the NP-complete PARTITION problem, which is: given $n$ positive rational numbers $\left(a_{1}, \ldots, a_{n}\right)$, is there a set $S$ such that $\sum_{i \in S} a_{i}=\sum_{i \notin S} a_{i}$ ? Consider an instance $I$ of PARTITION. Without loss of generality, suppose we have normalised the numbers so that $\sum_{i=1}^{n} a_{i}=1$. Then the problem instance sounds: Is there a set $S$ such that $\sum_{i \in S} a_{i}=\sum_{i \notin S} a_{i}=\frac{1}{2}$ ?

To construct the appropriate network we employ the networks given in Figure 1 and in Figure 2, where for each node $i \in\{1, \ldots, n\}$ we set $w_{i a}=w_{i b}=a_{i}$, and assume that the threshold of the nodes $a$ and $b$ is constant and equals $\frac{1}{2}$.

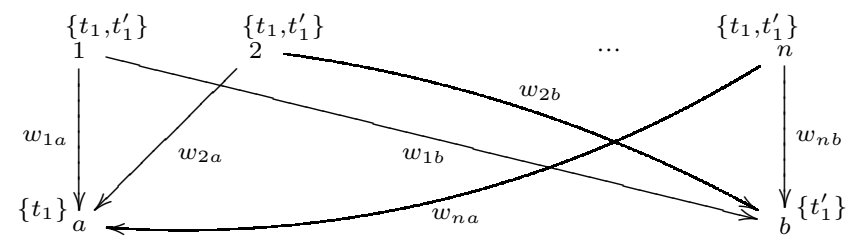

Figure 2: A network related to the PARTITION problem

We use two copies of the network given in Figure 1, one unchanged and the other in which the product $t_{1}$ is replaced by $t_{1}^{\prime}$, and construct the desired network $\mathcal{S}$ by identifying the node $a$ of the network from Figure 2 with the node 
marked by $\left\{t_{1}\right\}$ in the network from Figure 1 , and the node $b$ with the node marked by $\left\{t_{1}^{\prime}\right\}$ in the modified version of the network from Figure 1.

Suppose now that a solution to the considered instance of the PARTITION problem exists, that is, for some set $S \subseteq\{1, \ldots, n\}$ we have $\sum_{i \in S} a_{i}=\sum_{i \notin S} a_{i}=$ $\frac{1}{2}$. Consider the game $\mathcal{G}(\mathcal{S})$. Take the joint strategy formed by the following strategies:

- $t_{1}$ assigned to each node $i \in S$ in the network from Figure 2,

- $t_{1}^{\prime}$ assigned to each node $i \in\{1, \ldots, n\} \backslash S$ in the network from Figure 2,

- $t_{0}$ assigned to the nodes $a, b$ and the nodes 1 in both versions of the network from Figure 1,

- $t_{3}$ assigned to the nodes 2, 3 in both versions of the networks from Figure 1 and the two nodes marked by $\left\{t_{3}\right\}$,

- $t_{2}$ assigned to the nodes marked by $\left\{t_{2}\right\}$.

We claim that this joint strategy is a non-trivial Nash equilibrium. Consider first the player (i.e, node) $a$. The accumulated weight of its neighbours who chose strategy $t_{1}$ is $\frac{1}{2}$, so its payoff after switching to the strategy $t_{1}$ is 0 . Therefore $t_{0}$ is indeed a best response for player $a$. For the same reason, $t_{0}$ is also a best response for player $b$. The analysis for the other nodes is straightforward and left to the reader.

Conversely, suppose that a joint strategy $s$ is a Nash equilibrium in the game $\mathcal{G}(\mathcal{S})$. Then it is also a non-trivial Nash equilibrium since for each source node $i \in\{1, \ldots, n\}, s_{i} \neq t_{0}$. We claim that the strategy selected by the node $a$ in $s$ is $t_{0}$. Otherwise, this strategy equals $t_{1}$ and the strategies selected by the nodes of the network of Figure 1 form a Nash equilibrium in the game associated with this network. This yields a contradiction with our previous analysis of this network.

So $t_{0}$ is a best response of the node $a$ to the strategies of the other players chosen in $s$. This means that $\sum_{i \in\{1, \ldots, n\} \mid s_{i}=t_{1}} w_{i a} \leq \frac{1}{2}$. By the same reasoning $t_{0}$ is a best response of the node $b$ to the strategies of the other players chosen in $s$. This means that $\sum_{i \in\{1, \ldots, n\} \mid s_{i}=t_{1}^{\prime}} w_{i b} \leq \frac{1}{2}$.

But $\sum_{i=1}^{n} a_{i}=1$ and for $i \in\{1, \ldots, n\}, w_{i a}=w_{i b}=a_{i}$, and $s_{i} \in\left\{t_{1}, t_{1}^{\prime}\right\}$ since $i$ is a source node. So both above inequalities are in fact equalities. Consequently for $S:=\left\{i \in\{1, \ldots, n\} \mid s_{i}=t_{1}\right\}$ we have $\sum_{i \in S} a_{i}=\sum_{i \notin S} a_{i}$. In other words, there exists a solution to the considered instance of the PARTITION problem.

Finally, given an instance of the PARTITION problem, the above network $\mathcal{S}$ can be constructed from it in polynomial time. This proves the NP-hardness of the considered problem.

To prove that the problem lies in NP it suffices to notice that given a network $\mathcal{S}=(G, \mathcal{P}, P, \theta)$ with $n$ nodes checking whether a joint strategy is a non-trivial Nash equilibrium can be done by means of $n \cdot|\mathcal{P}|$ checks, so in polynomial time. 
Focussing on determined Nash equilibria does not change the matters.

Theorem 3. Deciding whether for a network $\mathcal{S}$ the game $\mathcal{G}(\mathcal{S})$ has a determined Nash equilibrium is NP-complete.

Proof. We use an instance of the PARTITION problem in the form of $n$ positive rational numbers $\left(a_{1}, \ldots, a_{n}\right)$, normalised as in the previous proof, and the network given in Figure 2.

Suppose now that a solution to the considered instance of the partition problem exists, that is for some set $S \subseteq\{1, \ldots, n\}$ we have $\sum_{i \in S} a_{i}=\sum_{i \notin S} a_{i}=$ $\frac{1}{2}$. Take the joint strategy $s$ formed by the following strategies:

- $t_{1}$ assigned to each node $i \in S$ and the node $a$,

- $t_{1}^{\prime}$ assigned to each node $i \in\{1, \ldots, n\} \backslash S$ and the node $b$.

Then $p_{a}\left(t_{1}, s_{-i}\right)=\frac{1}{2}-\theta\left(a, t_{1}\right)=0$, so $p_{a}\left(t_{1}, s_{-i}\right) \geq p_{a}\left(t_{0}, s_{-i}\right)$. Analogously $p_{b}\left(t_{1}^{\prime}, s_{-i}\right) \geq p_{b}\left(t_{0}, s_{-i}\right)$. So $s$ is a determined Nash equlibrium in the strategic game associated with the above network.

Consider now a determined Nash equilibrium $s$ in this game. Then $s_{a}=t_{1}$ and $p_{a}\left(t_{1}, s_{-i}\right) \geq p_{a}\left(t_{0}, s_{-i}\right)=0$. So for $S:=\left\{i \in\{1, \ldots, n\} \mid s_{i}=t_{1}\right\}$ we have $\sum_{i \in S} a_{i} \geq \frac{1}{2}$. Analogously $\sum_{i \notin S} a_{i} \geq \frac{1}{2}$. Since $\sum_{i=1}^{n} a_{i}=1$, in both cases we have in fact equalities and hence there exists a solution to the considered instance of the PARTITION problem.

Recall that the social network in Example 2 used three products. The following result shows that to construct a social network $\mathcal{S}$ such that $\mathcal{G}(\mathcal{S})$ has no Nash equilibrium in fact at least three products are required.

Theorem 4. For a network $\mathcal{S}$, if there exists a non-empty set $X \subseteq \mathcal{P}$ such that $|X| \leq 2$ and for all $i \in \operatorname{source}(\mathcal{S}), P(i) \cap X \neq \emptyset$ then $\mathcal{G}(\mathcal{S})$ has a Nash equilibrium and it can be computed in polynomial time.

In particular $\mathcal{G}(\mathcal{S})$ has a Nash equilibrium when all nodes $i$ have the same set of two products.

Proof. Given an initial joint strategy we call a maximal sequence of best response deviations to a given strategy $t$ (in an arbitrary order) a $t$-phase. Let $\mathcal{S}=(G, \mathcal{P}, P, \theta)$ where $G=(V, E, w)$.

First, suppose that $|X|=1$, say $X=\left\{t_{1}\right\}$. Let $s$ be the resulting joint strategy after performing a $t_{1}$-phase starting in the joint strategy $\overline{t_{0}}$. We show that $s$ is a Nash equilibrium. First note that $s_{j}=t_{1}$ for every $j \in \operatorname{source}(\mathcal{S})$. Further, in the $t_{1}$-phase, if a joint strategy $s^{2}$ is obtained from $s^{1}$ by having some nodes switch to product $t_{1}$ and $t_{1}$ is a best response for a node $i$ to $s_{-i}^{1}$, then $t_{1}$ remains a best response for $i$ to $s_{-i}^{2}$. Indeed, by the join the crowd property $p_{i}\left(t_{1}, s_{-i}^{2}\right) \geq p_{i}\left(t_{1}, s_{-i}^{1}\right)$ and $p_{i}\left(t_{1}, s_{-i}^{1}\right) \geq p_{i}\left(t_{0}, s_{-i}^{1}\right)$, so $p_{i}\left(t_{1}, s_{-i}^{2}\right) \geq p_{i}\left(t_{0}, s_{-i}^{2}\right)$. Consequently after the first $t_{1}$-phase, in the resulting joint strategy $s$, each node that has the strategy $t_{1}$ plays a best response. If for some $j, s_{j}=t_{0}$ then by 
the definition of the $t_{1}$-phase, $j$ is playing his best response as well. Therefore $s$ is a Nash equilibrium.

Now suppose that $|X|=2$, say $X=\left\{t_{1}, t_{2}\right\}$. Let $V_{t_{1}}=\left\{j \in \operatorname{source}(\mathcal{S}) \mid t_{1} \in\right.$ $P(j)\}$ and $\bar{V}_{t_{1}}=\left\{j \in \operatorname{source}(\mathcal{S}) \mid t_{1} \notin P(j)\right\}$. Let $\mathcal{S}^{t_{1}}=\left(G^{t_{1}}, \mathcal{P}, P, \theta\right)$, where $G^{t_{1}}$ is the induced subgraph of $G$ on the nodes $V \backslash \bar{V}_{t_{1}}$. Let $s^{t_{1}}$ be the resulting joint strategy in $\mathcal{S}^{t_{1}}$ after performing a $t_{1}$-phase starting in $\overline{t_{0}}$. By the previous argument, $s^{t_{1}}$ is a Nash equilibrium in $\mathcal{G}\left(\mathcal{S}^{t_{1}}\right)$. Now consider the joint strategy $s$ in $\mathcal{G}(\mathcal{S})$ defined as follows:

$$
s_{i}= \begin{cases}t_{0} & \text { if } i \in \bar{V}_{t_{1}} \\ s_{i}^{t_{1}} & \text { otherwise }\end{cases}
$$

Starting at $s$, we repeatedly perform a $t_{2}$-phase followed by a $t_{0}$-phase. We claim that this process terminates in a Nash equilibrium in $\mathcal{G}(\mathcal{S})$.

First note that if a joint strategy $s^{2}$ is obtained from $s^{1}$ by having some nodes switch to product $t_{2}$ and $t_{2}$ is a best response for a node $i$ to $s_{-i}^{1}$, then $t_{2}$ remains a best response for $i$ to $s_{-i}^{2}$. The argument is analogous to the one in the previous case. Therefore after the first $t_{2}$-phase each node that has the strategy $t_{2}$ plays a best response. Call the outcome of the first $t_{2}$-phase $s^{\prime \prime}$.

Now consider a node $i$ that deviated to $t_{0}$ starting at $s^{\prime \prime}$ by means of a best response. By the observation just made, node $i$ deviated from product $t_{1}$. So, again by the join the crowd property, this deviation does not affect the property that the nodes that selected $t_{2}$ in $s^{\prime \prime}$ play a best response. Iterating this reasoning we conclude that after the first $t_{0}$-phase each node that has the strategy $t_{2}$ continues to play a best response.

By the same reasoning subsequent $t_{2}$ and $t_{0}$-phases have the same effect on the set of nodes that have the strategy $t_{2}$, namely that each of these nodes continues to play a best response.

Moreover, this set continues to weakly increase. Consequently these repeated applications of the $t_{2}$-phase followed by the $t_{0}$-phase terminate, say in a joint strategy $s^{\prime}$. Now suppose that a node $i$ does not play a best response to $s_{-i}^{\prime}$. Then clearly $i \notin \operatorname{source}(\mathcal{S})$. If $s_{i}^{\prime}=t_{0}$, then by the construction $t_{2}$ is not a best response, so $t_{1}$ is a best response.

Suppose $s_{i}=t_{0}$. Consider the joint strategy $s^{t_{1}}$ which is a Nash equilibrium in $\mathcal{G}\left(\mathcal{S}^{t_{1}}\right)$. We have $p_{i}\left(t_{1}, s_{-i}^{t_{1}}\right) \leq p_{i}\left(t_{0}, s_{-i}^{t_{1}}\right)$. Since $i \notin \operatorname{source}(\mathcal{S})$, we have $s_{i}=s_{i}^{t_{1}}$. Since for all $j \in \bar{V}_{t_{1}}, t_{1} \notin P(j)$ we have $p_{i}\left(t_{1}, s_{-i}\right) \leq p_{i}\left(t_{0}, s_{-i}\right)$ as well. By the join the crowd property $p_{i}\left(t_{1}, s_{-i}^{\prime}\right) \leq p_{i}\left(t_{1}, s_{-i}\right)$, so $p_{i}\left(t_{1}, s_{-i}^{\prime}\right) \leq$ $p_{i}\left(t_{0}, s_{-i}^{\prime}\right)$, which yields a contradiction. Hence node $i$ deviated to $t_{0}$ from some intermediate joint strategy $s^{1}$ by selecting a best response. So $p_{i}\left(t_{1}, s_{-i}^{1}\right) \leq$ $p_{i}\left(t_{0}, s_{-i}^{1}\right)$. Moreover, by the join the crowd property $p_{i}\left(t_{1}, s_{-i}^{\prime}\right) \leq p_{i}\left(t_{1}, s_{-i}^{1}\right)$, so $p_{i}\left(t_{1}, s_{-i}^{\prime}\right) \leq p_{i}\left(t_{0}, s_{-i}^{\prime}\right)$, which yields a contradiction, as well.

Further, by the construction $s_{i}^{\prime} \neq t_{2}$, so the only alternative is that $s_{i}^{\prime}=t_{1}$. But then either $t_{0}$ or $t_{2}$ is a best response, which contradicts the construction of $s^{\prime}$. We conclude that $s^{\prime}$ is a Nash equilibrium in $\mathcal{G}(\mathcal{S})$.

Finally, note that each $t$-phase takes at most $n$ steps to terminate, where $n$ is the number of nodes in $G$. Since in each repeated iteration of a $t_{2}$-phase followed 
by a $t_{0}$-phase, the set of nodes which chose $t_{2}$ is weakly increasing, this process terminates after at most $\mathcal{O}(n)$ iterations. Therefore this Nash equilibrium can be computed in polynomial time.

\section{Polymatrix games}

We now apply the results of the previous section to a class of games that are more general than social network games.

A polymatrix game, see $[12,10]$, is a finite strategic game in which the influence of a pure strategy selected by any player on the payoff of any other player is always the same, regardless what strategies other players select. Formally, it is a game $\left(S_{1}, \ldots, S_{n}, p_{1}, \ldots, p_{n}\right)$ in which for all pairs of players $i$ and $j$ there exists a partial payoff function $a^{i j}$ such that for any joint strategy $s=\left(s_{1}, \ldots, s_{n}\right)$, the payoff of player $i$ is given by $p_{i}(s):=\sum_{j \neq i} a^{i j}\left(s_{i}, s_{j}\right)$.

Theorem 5. Deciding whether a polymatrix game has a Nash equilibrium is NP-complete.

Proof. It is clear that the problem is in NP since one can guess a joint strategy and check whether it is a Nash equilibrium in polynomial time. To prove the hardness result we show that social network games are a subclass of polymatrix games. The claim then follows from Theorem 1.

For a social network $\mathcal{S}=(G, \mathcal{P}, P, \theta)$ with $n$ agents, let the associated game be $\mathcal{G}(\mathcal{S})=\left(S_{1}, \ldots, S_{n}, p_{1}, \ldots, p_{n}\right)$. We translate $\mathcal{G}(\mathcal{S})$ into a payoff equivalent polymatrix game $\mathcal{G}^{\prime}=\left(S_{1}, \ldots, S_{n}, p_{1}^{\prime}, \ldots, p_{n}^{\prime}\right)$ as follows. For a joint strategy $s$ and players $i$ and $j$, the partial payoff functions are given by

- for $i \in \operatorname{source}(\mathcal{S})$,

$$
a^{i j}\left(s_{i}, s_{j}\right):= \begin{cases}0 & \text { if } s_{i}=t_{0}, \\ \frac{c_{0}}{n-1} & \text { if } s_{i} \in P(i),\end{cases}
$$

- for $i \notin \operatorname{source}(\mathcal{S})$,

$$
a^{i j}\left(s_{i}, s_{j}\right):= \begin{cases}0 & \text { if } s_{i}=t_{0}, \\ w_{j i}-\frac{\theta\left(i, s_{i}\right)}{n-1} & \text { if } j \in N(i), s_{i} \neq t_{0} \text { and } s_{i}=s_{j}, \\ -\frac{\theta\left(i, s_{i}\right)}{n-1} & \text { otherwise. }\end{cases}
$$

The payoff function for player $i$ is then defined as $p_{i}^{\prime}(s)=\sum_{j \neq i} a^{i j}\left(s_{i}, s_{j}\right)$. To complete the proof, we show that for all $i \in\{1, \ldots, n\}$, for all joint strategies $s$, we have $p_{i}^{\prime}(s)=p_{i}(s)$. Recall that for a player $i$ and a joint strategy $s$, $\mathcal{N}_{i}^{s_{i}}(s)=\left\{j \in N(i) \mid s_{i}=s_{j}\right\}$. We have the following cases:

- $s_{i}=t_{0}$. Then $p_{i}^{\prime}(s)=0=p_{i}(s)$.

- $s_{i} \neq t_{0}$ and $i \in \operatorname{source}(\mathcal{S})$.

Then $p_{i}^{\prime}(s)=\sum_{j \neq i} \frac{c_{0}}{n-1}=(n-1) \cdot \frac{c_{0}}{n-1}=p_{i}(s)$. 
- $s_{i} \neq t_{0}$ and $i \notin \operatorname{source}(\mathcal{S})$.

$$
\text { Then } \begin{aligned}
p_{i}^{\prime}(s) & =\sum_{j \in \mathcal{N}_{i}^{s_{i}}(s)}\left(w_{j i}-\frac{\theta\left(i, s_{i}\right)}{n-1}\right)+\sum_{j \neq i, j \notin \mathcal{N}_{i}^{s_{i}}(s)}-\frac{\theta\left(i, s_{i}\right)}{n-1} \\
& =\sum_{j \in \mathcal{N}_{i}^{s_{i}}(s)} w_{j i}-(n-1) \cdot \frac{\theta\left(i, s_{i}\right)}{n-1} \\
& =p_{i}(s) .
\end{aligned}
$$

\section{Nash equilibria: special cases}

In view of the fact that in general Nash equilibria may not exist we now consider networks with special properties of the underlying directed graph. We focus on three natural classes.

\subsection{Directed acyclic graphs}

We consider first networks whose underlying graph is a directed acyclic graph (DAG). Intuitively, such networks correspond to hierarchical organizations. This restriction leads to a different outcome in the analysis of Nash equilibria.

Given a DAG $G:=(V, E)$, we use a fixed level by level enumeration $\operatorname{rank}()$ of its nodes so that for all $i, j \in V$

$$
\text { if } \operatorname{rank}(i)<\operatorname{rank}(j) \text {, then there is no path in } G \text { from } j \text { to } i \text {. }
$$

Theorem 6. Consider a network $\mathcal{S}$ whose underlying graph is a DAG.

(i) $\mathcal{G}(\mathcal{S})$ always has a non-trivial Nash equilibrium.

(ii) Deciding whether $\mathcal{G}(\mathcal{S})$ has a determined Nash equilibrium is NP-complete.

Proof.

(i) We proceed by assigning to each node a strategy following the order determined by (1). Given a node we assign to it a best response to the sequence of strategies already assigned to all his neighbours. (By definition the strategies of other players have no influence on the choice of a best response.) This yields a non-trivial Nash equilibrium. This result is also an immediate consequence of Theorem 23 proved in Section 8.

(ii) The claim follows from Theorem 3 since the underlying graph of the network from Figure 2 used in its proof is a DAG.

Note that when the underlying graph is a DAG all Nash equilibria are nontrivial. Further, in the procedure described in $(i)$, in general more than one best response can exist. In that case multiple Nash equilibria exist.

The above procedure uses the set of best responses $B R_{i}\left(s_{N(i)}\right)$ of player $i$ to the joint strategy $s_{N(i)}$ of his neighbours in $\mathcal{G}(\mathcal{S})$. This set is defined directly in terms of $s_{N(i)}$ and $\mathcal{S}$ as follows, where $\mathcal{S}=(G, \mathcal{P}, P, \theta)$. 
Let

$$
\begin{aligned}
& Z_{i}^{>0}\left(s_{N(i)}\right):=\left\{t \in P(i) \mid \sum_{k \in N(i), s_{k}=t} w_{k i}-\theta(i, t)>0\right\}, \\
& Z_{i}^{=0}\left(s_{N(i)}\right):=\left\{t_{0}\right\} \cup\left\{t \in P(i) \mid \sum_{k \in N(i), s_{k}=t} w_{k i}-\theta(i, t)=0\right\} .
\end{aligned}
$$

Then

$$
B R_{i}\left(s_{N(i)}\right):= \begin{cases}\underset{t \in Z_{i}^{>0}\left(s_{N(i)}\right)}{\operatorname{argmax}}\left(\sum_{k \in N(i), s_{k}=t} w_{k i}-\theta(i, t)\right) & \text { if } Z_{i}^{>0}\left(s_{N(i)}\right) \neq \emptyset \\ Z_{i}^{=0}\left(s_{N(i)}\right) & \text { otherwise }\end{cases}
$$

Finally, we consider the price of anarchy and the price of stability for the considered class of games. The following simple result holds.

Theorem 7. The price of anarchy and the price of stability for the games associated with the networks whose underlying graph is a DAG is unbounded.

Proof. Consider the network depicted in Figure 3.

$$
i \stackrel{\left\{t_{1}\right\} w_{i j}}{\longrightarrow} j^{\left\{t_{2}\right\} w_{j k}} \longrightarrow k^{\left\{t_{2}\right\}}
$$

Figure 3: A network with a high price of anarchy and stability

Choose an arbitrary value $r>0$. Suppose first that there exists weights and thresholds such that $w_{j k}-\left(\theta\left(j, t_{2}\right)+\theta\left(k, t_{2}\right)\right)>r c_{0}$. (Recall that the payoff of player $i$ is $c_{0}$.)

The game associated with this network has a unique Nash equilibrium, namely the joint strategy $\left(t_{1}, t_{0}, t_{0}\right)$ assigned to the sequence $(i, j, k)$ of nodes. Its social welfare is $c_{0}$. In contrast, the social optimum is achieved by the joint strategy $\left(t_{1}, t_{2}, t_{2}\right)$ and equals

$$
c_{0}+w_{j k}-\left(\theta\left(j, t_{2}\right)+\theta\left(k, t_{2}\right)\right)>r c_{0} .
$$

So for every value $r>0$ there is a network whose game has price of anarchy and price of stability higher than $r$.

Suppose now that the inequality $w_{j k}-\left(\theta\left(j, t_{2}\right)+\theta\left(k, t_{2}\right)\right)>r c_{0}$ does not hold for any choice of weights and thresholds. (This is for instance the case when $r c_{0} \geq 1$, which can be the case as $c_{0}$ and $r$ are arbitrary.) In that case, we modify the above social network as follows. First, we replace the node $k$ by $\left\lceil r c_{0}+1\right\rceil$ nodes, all direct descendants of node $j$ and each with the product set $\left\{t_{2}\right\}$. Then we choose the weights and the thresholds in such a way that the sum of all these weights minus the sum of all the thresholds for the product $t_{2}$ exceeds $r c_{0}$. In the resulting game, by the same argument as above, both the price of anarchy and price of stability are higher than $r$. 


\subsection{Simple cycles}

Next, we consider networks whose underlying graph is a simple cycle. To fix the notation suppose that the underlying graph is $1 \rightarrow 2 \rightarrow \ldots \rightarrow n \rightarrow 1$. We assume that the counting is done in cyclic order within $\{1, \ldots, n\}$ using the increment operation $i \oplus 1$ and the decrement operation $i \ominus 1$. In particular, $n \oplus 1=1$ and $1 \ominus 1=n$. The payoff functions can then be rewritten as follows:

$$
p_{i}(s):= \begin{cases}0 & \text { if } s_{i}=t_{0} \\ w_{i \ominus 1}-\theta\left(i, s_{i}\right) & \text { if } s_{i}=s_{i \ominus 1} \text { and } s_{i} \in P(i) \\ -\theta\left(i, s_{i}\right) & \text { otherwise }\end{cases}
$$

Clearly $\overline{t_{0}}$ is a trivial Nash equilibrium. The following observation clarifies when other Nash equilibria exist.

Theorem 8. Consider a network $\mathcal{S}$ whose underlying graph is a simple cycle. Then $s$ is a non-trivial (respectively, determined) Nash equilibrium of the game $\mathcal{G}(\mathcal{S})$ iff $s$ is of the form $\bar{t}$ for some product $t$ and for all $i, p_{i}(s) \geq 0$.

Proof. $(\Rightarrow)$ Consider a non-trival Nash equilibrium $s$. Suppose that $s_{i}=t$ for a product $t$. We have $p_{i}(s) \geq p_{i}\left(t_{0}, s_{-i}\right)=0$, so $s_{i \ominus 1}=t$. Iterating this reasoning we conclude that $s=\bar{t}$.

$(\Leftarrow)$ Straightforward.

This yields a straightforward algorithm that allows us to check whether there exists a non-trivial, respectively, determined, Nash equilibrium.

Theorem 9. Consider a network $\mathcal{S}=(G, \mathcal{P}, P, \theta)$ whose underlying graph is a simple cycle. There is a procedure that runs in time $O(|\mathcal{P}| \cdot n)$, where $n$ is the number of nodes in $G$, that decides whether $\mathcal{G}(\mathcal{S})$ has a non-trivial (respectively, determined) Nash equilibrium.

Proof. Thanks to Theorem 8 we can use the following procedure.

$$
\operatorname{VerifyNashCycle}(\mathcal{S})
$$

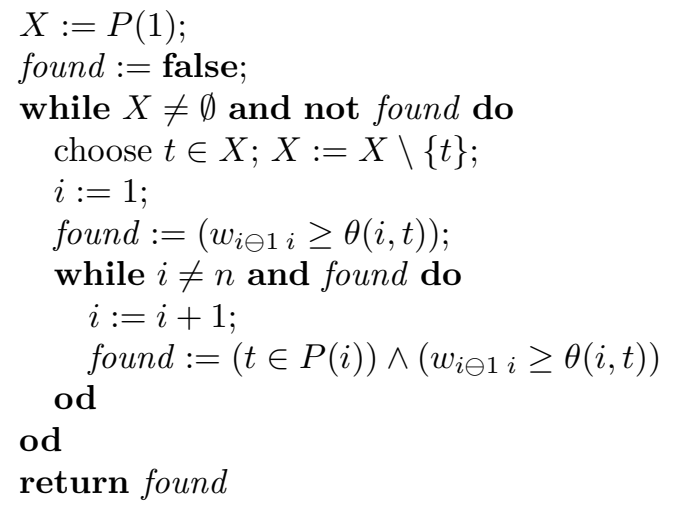

This procedure returns true if a non-trivial (or equivalently, a determined) Nash equilibrium exists and false otherwise. Its running time is $O(|\mathcal{P}| \cdot n)$. 
Next, we consider the price of anarchy and the price of stability. We have the following counterpart of Theorem 7 .

Theorem 10. The price of anarchy and the price of stability for the games associated with the networks whose underlying graph is a simple cycle is unbounded.

Proof. Choose an arbitrary value $r>0$ and let $\epsilon$ be such that $\epsilon<\min \left(\frac{1}{4}, \frac{1}{2(r+1)}\right)$. Then both $1-2 \epsilon>2 \epsilon$ and $\frac{1-2 \epsilon}{2 \epsilon}>r$.

Consider the network depicted in Figure 4.

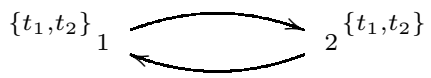

Figure 4: Another network with a high price of anarchy and stability

We assume that

$w_{12}-\theta\left(2, t_{2}\right)=1-\epsilon, w_{21}-\theta\left(1, t_{2}\right)=-\epsilon, w_{12}-\theta\left(2, t_{1}\right)=\epsilon, w_{21}-\theta\left(1, t_{1}\right)=\epsilon$.

Then the social optimum is achieved in the joint strategy $\left(t_{2}, t_{2}\right)$ and equals $1-2 \epsilon$. There are two Nash equilibria, $\left(t_{1}, t_{1}\right)$ and the trivial one, with the respective social welfare $2 \epsilon$ and 0 .

In the case of the price of anarchy we deal with the division by zero. We interpret the outcome as $\infty$. The price of stability equals $\frac{1-2 \epsilon}{2 \epsilon}$, so is higher than $r$.

\subsection{Graphs with no source nodes}

Finally, we consider the case when the underlying graph $G=(V, E)$ of a network $\mathcal{S}$ has no source nodes, i.e., for all $i \in V, N(i) \neq \emptyset$. Intuitively, such a network corresponds to a 'circle of friends': everybody has a friend (a neighbour). For such networks we prove the following result.

Theorem 11. Consider a network $\mathcal{S}=(G, \mathcal{P}, P, \theta)$ whose underlying graph has no source nodes. There is a procedure that runs in time $O\left(|\mathcal{P}| \cdot n^{3}\right)$, where $n$ is the number of nodes in $G$, that decides whether $\mathcal{G}(\mathcal{S})$ has a non-trivial Nash equilibrium.

The proof of Theorem 11 requires some characterization results that are of independent interest. The following concept plays a crucial role. Here and elsewhere we only consider subgraphs that are induced and identify each such subgraph with its set of nodes. (Recall that $\left(V^{\prime}, E^{\prime}\right)$ is an induced subgraph of $(V, E)$ if $V^{\prime} \subseteq V$ and $E^{\prime}=E \cap\left(V^{\prime} \times V^{\prime}\right)$.)

We say that a (non-empty) strongly connected subgraph (in short, SCS) $C_{t}$ of $G$ is self-sustaining for a product $t$ if for all $i \in C_{t}$,

- $t \in P(i)$, 
- $\sum_{j \in N(i) \cap C_{t}} w_{j i} \geq \theta(i, t)$.

An easy observation is that if $\mathcal{S}$ is a network with no source nodes, then it always has a trivial Nash equilibrium, $\overline{t_{0}}$. The following lemma states that for such networks every non-trivial Nash equilibrium satisfies a structural property which relates it to the set of self- sustaining SCSs in the underlying graph. We use the following notation: for a joint strategy $s$ and a product $t, \mathcal{A}_{t}(s):=\{i \in$ $\left.V \mid s_{i}=t\right\}$ and $P(s):=\left\{t \mid \exists i \in V\right.$ with $\left.s_{i}=t\right\}$.

Lemma 12. Let $\mathcal{S}=(G, \mathcal{P}, P, \theta)$ be a network whose underlying graph has no source nodes. If $s \neq \bar{t}_{0}$ is a Nash equilibrium in $\mathcal{G}(\mathcal{S})$, then for all products $t \in P(s) \backslash\left\{t_{0}\right\}$ and $i \in \mathcal{A}_{t}(s)$ there exists a self-sustaining $S C S C_{t} \subseteq \mathcal{A}_{t}(s)$ for $t$ and $a j \in C_{t}$ such that $j \rightarrow^{*} i$.

Proof. Suppose $s \neq \overline{t_{0}}$ is a Nash equilibrium. Take any product $t \neq t_{0}$ and an agent $i$ such that $s_{i}=t$ (by assumption, at least one such $t$ and $i$ exists). Recall that $\mathcal{N}_{i}^{t}(s)$ is the set of neighbours of $i$ who adopted the product $t$ in $s$. Consider the set of nodes Pred $:=\bigcup_{m \in \mathbb{N}} \operatorname{Pred}_{m}$, where

- $\operatorname{Pred}_{0}:=\{i\}$,

- $\operatorname{Pred}_{m+1}:=\operatorname{Pred}_{m} \cup \bigcup_{j \in \operatorname{Pred}_{m}} \mathcal{N}_{j}^{t}(s)$.

By construction for all $j \in$ Pred, $s_{j}=t$ and $\mathcal{N}_{j}^{t}(s) \subseteq$ Pred. Moreover, since $s$ is a Nash equilibrium, we also have $\sum_{k \in \mathcal{N}_{j}^{t}(s)} w_{k j} \geq \theta(j, t)$.

Consider the partial ordering $<$ between the strongly connected components of the graph induced by Pred defined by: $C<C^{\prime}$ iff $j \rightarrow k$ for some $j \in C$ and $k \in C^{\prime}$. Now take some SCS $C_{t}$ induced by a strongly connected component that is minimal in the $<$ ordering. Then for all $k \in C_{t}$ we have $\mathcal{N}_{k}^{t}(s) \subseteq C_{t}$ and hence $\mathcal{N}_{k}^{t}(s) \subseteq N(k) \cap C_{t}$. This shows that $C_{t}$ is self-sustaining.

Moreover, by the construction of Pred for all $j \in$ Pred, and a fortiori for all $j \in C_{t}$, we also have $j \rightarrow^{*} i$. Since the choice of $t$ and $i$ was arbitrary, the claim follows.

The converse of Lemma 12 does not hold. Indeed, consider the equitable network given in Figure 5. The product set of each agent is marked next to the node denoting the agent. Assume that the threshold of each node is a constant smaller than $\frac{1}{k}$, where $k$ is the number of incoming edges. So each agent has a non-negative payoff when he adopts any product adopted by some of his neighbours. Consider the joint strategy $s$ in which agents 1,2 and 3 adopt product $t_{1}$ and agents 4,5 and 6 adopt product $t_{3}$, i.e., $s=\left(t_{1}, t_{1}, t_{1}, t_{3}, t_{3}, t_{3}\right)$. It follows from the definition that $s$ satisfies the following condition of Lemma 12 :

- for all products $t \in P(s) \backslash\left\{t_{0}\right\}$ and $k \in \mathcal{A}_{t}(s)$ there exists a self-sustaining SCS $C_{t} \subseteq \mathcal{A}_{t}(s)$ for $t$ and $j \in S_{t}$ such that $j \rightarrow^{*} k$. 


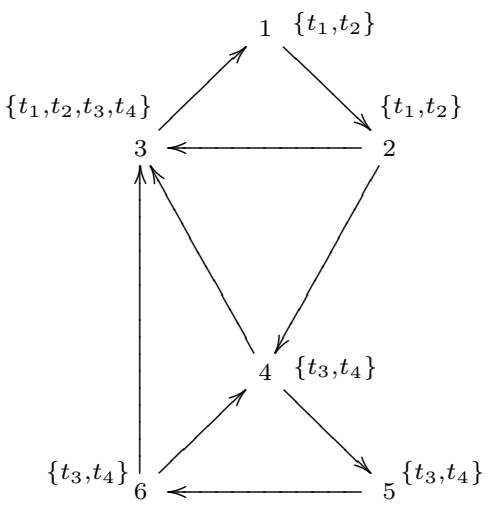

Figure 5: An equitable network

However, $s$ is not a Nash equilibrium since agent 3 has the incentive to deviate to product $t_{3}$. Also, note that the joint strategy $s^{\prime}=\left(t_{0}, t_{0}, t_{3}, t_{3}, t_{3}, t_{3}\right)$ is a Nash equilibrium.

Using Lemma 12, we can now provide a necessary and sufficient condition for the existence of non-trivial Nash equilibria for the considered networks.

Lemma 13. Let $\mathcal{S}=(G, \mathcal{P}, P, \theta)$ be a network whose underlying graph has no source nodes. The joint strategy $\overline{t_{0}}$ is a unique Nash equilibrium in $\mathcal{G}(\mathcal{S})$ iff there does not exist a product $t$ and a self-sustaining $S C S C_{t}$ for $t$ in $G$.

Proof. $(\Leftarrow)$ By Lemma 12 .

$(\Rightarrow)$ Suppose there exists a self-sustaining SCS $C_{t}$ for a product $t$. Let $R$ be the set of nodes reachable from $C_{t}$ which eventually can adopt product $t$. Formally, $R:=\bigcup_{m \in \mathbb{N}} R_{m}$ where

- $R_{0}:=C_{t}$,

- $R_{m+1}:=R_{m} \cup\left\{j \mid t \in P(j)\right.$ and $\left.\sum_{k \in N(j) \cap R_{m}} w_{k j} \geq \theta(j, t)\right\}$.

Let $s$ be the joint strategy such that for all $j \in R$, we have $s_{j}=t$ and for all $k \in V \backslash R$, we have $s_{k}=t_{0}$. It follows directly from the definition of $R$ that $s$ satisfies the following properties:

(P1) for all $i \in V, s_{i}=t_{0}$ or $s_{i}=t$,

(P2) for all $i \in V, s_{i} \neq t_{0}$ iff $i \in R$,

(P3) for all $i \in V$, if $i \in R$ then $p_{i}(s) \geq 0$.

We show that $s$ is a Nash equilibrium. Consider first any $j$ such that $s_{j}=t$ (so $\left.s_{j} \neq t_{0}\right)$. By $(\mathrm{P} 2) j \in R$ and by $(\mathrm{P} 3) p_{j}(s) \geq 0$. Since $p_{j}\left(s_{-j}, t_{0}\right)=$ $0 \leq p_{j}(s)$, player $j$ does not gain by deviating to $t_{0}$. Further, by $(\mathrm{P} 1)$, for all $k \in N(j), s_{k}=t$ or $s_{k}=t_{0}$ and therefore for all products $t^{\prime} \neq t$ we have 
$p_{j}\left(s_{-j}, t^{\prime}\right)<0 \leq p_{j}(s)$. Thus player $j$ does not gain by deviating to any product $t^{\prime} \neq t$ either.

Next, consider any $j$ such that $s_{j}=t_{0}$. We have $p_{j}(s)=0$ and from (P2) it follows that $j \notin R$. By the definition of $R$ we have $\sum_{k \in N(j) \cap R} w_{k j}<\theta(j, t)$. Thus $p_{j}\left(s_{-j}, t\right)<0$. Moreover, for all products $t^{\prime} \neq t$ we also have $p_{j}\left(s_{-j}, t^{\prime}\right)<0$ for the same reason as above. So player $j$ does not gain by a unilateral deviation. We conclude that $s$ is a Nash equilibrium.

Next, for a product $t \in \mathcal{P}$, we define the set $X_{t}:=\bigcap_{m \in \mathbb{N}} X_{t}^{m}$, where

- $X_{t}^{0}:=\{i \in V \mid t \in P(i)\}$,

- $X_{t}^{m+1}:=\left\{i \in V \mid \sum_{j \in N(i) \cap X_{t}^{m}} w_{j i} \geq \theta(i, t)\right\}$.

We need the following characterization result.

Lemma 14. Let $\mathcal{S}$ be a network whose underlying graph has no source nodes. There exists a non-trivial Nash equilibrium in $\mathcal{G}(\mathcal{S})$ iff there exists a product $t$ such that $X_{t} \neq \emptyset$.

Proof. Suppose $\mathcal{S}=(G, \mathcal{P}, P, \theta)$.

$(\Rightarrow)$ It follows directly from the definitions that if there is a self-sustaining SCS $C_{t}$ for product $t$, then $C_{t} \subseteq X_{t}$. Suppose now that for all $t, X_{t}=\emptyset$. Then for all $t$, there is no self-sustaining SCS for $t$. So by Lemma $13, \overline{t_{0}}$ is a unique Nash equilibrium.

$(\Leftarrow)$ Suppose there exists $t$ such that $X_{t} \neq \emptyset$. Let $s$ be the joint strategy defined as follows:

$$
s_{i}:= \begin{cases}t & \text { if } i \in X_{t} \\ t_{0} & \text { if } i \notin X_{t}\end{cases}
$$

By the definition of $X_{t}$, for all $i \in X_{t}, p_{i}(s) \geq 0$. So no player $i \in X_{t}$ gains by deviating to $t_{0}$ (as then his payoff would become 0 ) or to a product $t^{\prime} \neq t$ (as then his payoff would become negative since no player adopted $t^{\prime}$ ). Also, by the definition of $X_{t}$ and of the joint strategy $s$, for all $i \notin X_{t}$ and for all $t^{\prime} \in P(i)$, $p_{i}\left(t^{\prime}, s_{-i}\right)<0$. Therefore, no player $i \notin X_{t}$ gains by deviating to a product $t^{\prime}$ either. It follows that $s$ is a Nash equilibrium.

This theorem leads to a direct proof of the claimed result.

Proof of Theorem 11.

We use the following procedure for checking for the existence of a non-trivial Nash equilibrium.

$$
\operatorname{VerifyNash}(\mathcal{S})
$$

found $:=$ false;

while $\mathcal{P} \neq \emptyset$ and $\neg$ found do

choose $t \in \mathcal{P}$;

$\mathcal{P}:=\mathcal{P}-\{t\}$ 


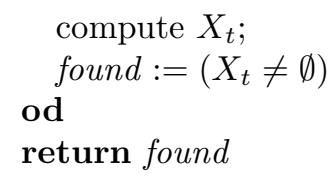

On the account of Lemma 14 this procedure returns true if a non-trivial Nash equilibrium exists and false otherwise. To assess its complexity, note that for a network $\mathcal{S}=(G, \mathcal{P}, P, \theta)$ and a fixed product $t$, the set $X_{t}$ can be constructed in time $O\left(n^{3}\right)$, where $n$ is the number of nodes in $G$. Indeed, each iteration of $X_{t}^{m}$ requires at most $O\left(n^{2}\right)$ comparisons and the fixed point is reached after at most $n$ steps. In the worst case, we need to compute $X_{t}$ for every $t \in \mathcal{P}$, so the procedure runs in time $O\left(|\mathcal{P}| \cdot n^{3}\right)$.

In fact, the proof of Lemma 14 shows that if a non-trivial Nash equilibrium exists, then it can be constructed in polynomial time as well. The complexity changes in the case of determined Nash equilibria.

Theorem 15. For a network $\mathcal{S}$ whose underlying graph has no source nodes, deciding whether the game $\mathcal{G}(\mathcal{S})$ has a determined Nash equilibrium is NPcomplete.

Proof. We modify the network given in Figure 2 so that the graph has no source nodes. To this end we 'twin' each node $i \in\{1, \ldots, n\}$ with a new node $i^{\prime}$, also with the product set $\left\{t_{1}, t_{1}^{\prime}\right\}$, by adding edges $\left(i, i^{\prime}\right)$ and $\left(i^{\prime}, i\right)$. Additionally, we choose the weights $w_{i i^{\prime}}$ and $w_{i^{\prime} i}$ and the corresponding thresholds so that when $i$ and $i^{\prime}$ adopt a common product, their payoff is positive.

For the so modified network we can now repeat the proof of Theorem 3.

\section{Finite best response property: general case}

We noted already that some social network games do not have a Nash equilibrium. A fortiori, such games do not have the finite best response property (FBRP) defined in Subsection 2.1. We now analyze the complexity of determining whether a game has the FBRP. We establish the following general result.

Theorem 16. Deciding whether for a network $\mathcal{S}$ the game $\mathcal{G}(\mathcal{S})$ has the FBRP is co-NP-hard.

Proof. We prove that the complement of the problem is NP-hard. We use again an instance of the PARTITION problem in the form of $n$ positive rational numbers $\left(a_{1}, \ldots, a_{n}\right)$, appropriately normalized, so that (this time) $\sum_{i=1}^{n} a_{i}=\frac{1}{2}$, and the network given in Figure 6 . For every node in the network, the product set is $\left\{t_{1}, t_{2}\right\}$ and for $i \in\{1, \ldots, n\}$ we set $w_{i a}=w_{i b}=a_{i}$. The other three weights are $\frac{1}{2}$. Next, we set $\theta\left(a, t_{1}\right)=\theta\left(b, t_{2}\right)=\frac{3}{4}$ and stipulate that for other inputs $\theta$ yields $\frac{1}{2}$.

Suppose now that a solution to the considered instance of the PARTITION problem exists, that is for some set $S \subseteq\{1, \ldots, n\}$ we have $\sum_{i \in S} a_{i}=\sum_{i \notin S} a_{i}=$ 


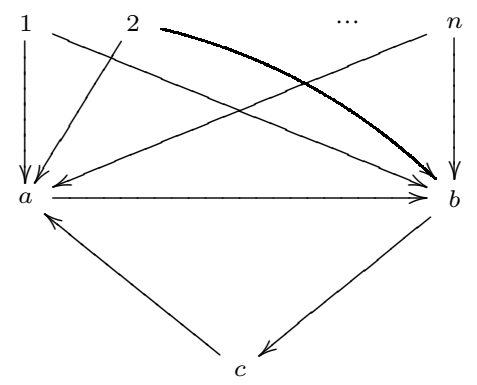

Figure 6: A network related to the FBRP

$\frac{1}{4}$. Consider the game $\mathcal{G}(\mathcal{S})$. Assume now that strategy $t_{1}$ is selected by each node $i \in S$ and strategy $t_{2}$ by each node $i \in\{1, \ldots, n\} \backslash S$. Identify each completion of this selection to a strategy profile with the triple of strategies selected by the nodes $a, b$ and $c$. Then it is easy to check that Figure 7 exhibits an infinite best response improvement path in $\mathcal{G}(\mathcal{S})$.

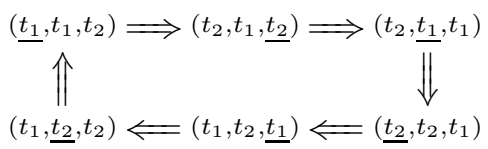

Figure 7: An infinite best response improvement path

Suppose that an infinite best response improvement path $\xi$ exists in $\mathcal{G}(\mathcal{S})$. The payoff for the node $c$ is always at most 0 . Hence $t_{0}$ is always a best response for $c$, so it is never chosen by $c$ in $\xi$. This means that in $\xi$, the node $c$ alternates between the strategies $t_{1}$ and $t_{2}$. Hence strategies $t_{1}$ and $t_{2}$ are also infinitely often selected in $\xi$ by the nodes $a$ and $b$. Consequently, the payoff for the node $a$ at the moment it switches to $t_{1}$ has to be $\geq 0$ and the payoff for the node $b$ at the moment it switches to $t_{2}$ has to be $\geq 0$. This means that $\sum_{i \in\{1, \ldots, n\} \mid s_{i}=t_{1}} w_{i a} \geq \frac{1}{4}$ and $\sum_{i \in\{1, \ldots, n\} \mid s_{i}=t_{2}} w_{i b} \geq \frac{1}{4}$. But $\sum_{i=1}^{n} a_{i}=\frac{1}{2}$ and for $i \in\{1, \ldots, n\}, w_{i a}=w_{i b}=a_{i}$, and $s_{i} \in\left\{t_{1}, t_{2}\right\}$. So both above inequalities are in fact equalities. Consequently there exists a solution to the considered instance of the PARTITION problem.

It would be interesting to find out precisely the complexity of determining whether a social network game has the FBRP. We conjecture that it is not co-NP. 


\section{Finite best response improvement property: special cases}

As in the case of Nash equilibria we now analyze the FBRP for social network games whose underlying graph satisfies certain properties.

\subsection{Directed acyclic graphs}

We begin with social network games whose underlying graph is a DAG. Then the following positive result holds.

Theorem 17. Consider a network $\mathcal{S}$ whose underlying graph is a DAG. Then the game $\mathcal{G}(\mathcal{S})$ has the FBRP.

This is a direct consequence of a stronger result, Theorem 23 of Section 9.1.

\subsection{Simple cycles}

The property that the game has the FBRP does not hold anymore when the underlying graph is a simple cycle. To see this consider Figure 8(a). Suppose that $\bar{t}$ is a Nash equilibrium in which each player gets a strictly positive payoff. Figure $8(\mathrm{~b})$ then shows an infinite best response improvement path. In each strategy profile, we underline the strategy that is not a best response to the choice of other players.

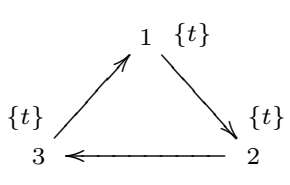

(a)

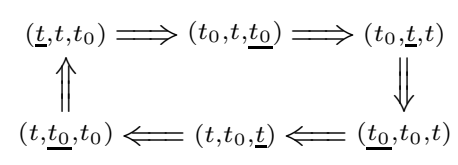

(b)

Figure 8: A network with an infinite best response improvement path

Next consider Figure 9(a). Suppose that both $\overline{t_{1}}$ and $\overline{t_{2}}$ are Nash equilibria. Then Figure 9(b) shows an infinite best response improvement path. This essentially repeats the reasoning used to show that the network given in Figure 6 has an infinite best response improvement path.

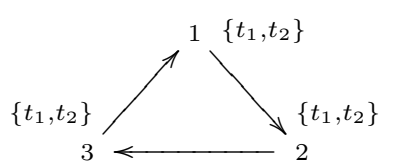

(a)

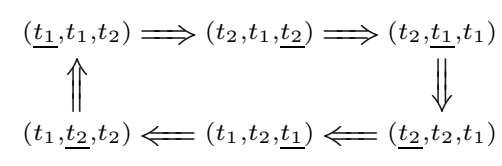

(b)

Figure 9: Another network with an infinite best response improvement path 
One can easily generalize the above two examples to simple cycles with more than three nodes. Below we show that when the game does not have the FBRP, the network is necessarily of one of the above two types. This will allow us to establish the following complexity result concerning the FBRP property.

Theorem 18. Consider a network $\mathcal{S}=(G, \mathcal{P}, P, \theta)$ such that $G$ is a simple cycle. There is a procedure that runs in time $O(|\mathcal{P}| \cdot n)$, where $n$ is the number of nodes in $G$, that decides whether the game $\mathcal{G}(\mathcal{S})$ has the FBRP.

We need the following characterization result.

Theorem 19. Let $\mathcal{S}$ be a network whose underlying graph is a simple cycle.

(i) Suppose that $\mathcal{S}$ has 2 nodes. Then the game $\mathcal{G}(\mathcal{S})$ has the FBRP.

(ii) Suppose that $\mathcal{S}$ has at least 3 nodes. Then the game $\mathcal{G}(\mathcal{S})$ does not have the FBRP iff either it has a determined Nash equilibrium s such that for all $i, p_{i}(s)>0$ or it has two determined Nash equilibria.

Proof. (i) A simple analysis, which we leave to the reader, shows that the longest possible improvement path is of length five and is of the form $\left(t_{1}, t_{2}\right),\left(t_{0}, t_{2}\right)$, $\left(t_{2}, t_{2}\right),\left(t_{2}, t_{0}\right),\left(t_{0}, t_{0}\right)$.

(ii) $(\Rightarrow)$ Consider an infinite best response improvement path $\xi$. Some node changes his strategy in $\xi$ infinitely often. This means that some node, say $i$, selects in $\xi$ some product $t$ infinitely often. Indeed, otherwise from some moment on in each strategy profile in $\xi$ its strategy would be $t_{0}$, which is not the case.

Each time node $i$ switches in $\xi$ to the product $t$, it selects a best response, so its payoff becomes at least 0 . Consequently, at the moment of such a switch its predecessor $i \ominus 1$ 's strategy is necessarily $t$ as well. So if from some moment on node $i \ominus 1$ does not switch from the strategy $t$, then node $i$ does not switch from $t$ either. This shows that node $i \ominus 1$ also selects in $\xi$ product $t$ infinitely often. Iterating this reasoning we conclude that each node selects in $\xi$ the product $t$ infinitely often. Therefore, for all $i, t \in P(i)$. Since the payoff of $i$ depends only on the choice of $i \ominus 1$, we also have that $p_{i}(\bar{t}) \geq 0$ for all $i$. By Theorem $8, \bar{t}$ is a Nash equilibrium.

This shows that if a node selects in $\xi$ some product $t_{1}$ infinitely often, then all nodes select in $\xi$ the product $t_{1}$ infinitely often and $\overline{t_{1}}$ is a Nash equilibrium. Suppose now that $\bar{t}$ is a unique determined Nash equilibrium. This means that all other products are selected in $\xi$ finitely often. So from some moment on in $\xi$ nodes select only $t$ or $t_{0}$. In this suffix $\eta$ of $\xi$ each node selects $t$ infinitely often. Further, each switch to $t$ from $t_{0}$ is a better response. Hence each time a node switches in $\eta$ to $t$ its payoff becomes $>0$. This shows that $\bar{t}$ is a determined Nash equilibrium such that for all $i, p_{i}(\bar{t})>0$.

$(\Leftarrow)$ Suppose first that the game $\mathcal{G}(\mathcal{S})$ has a determined Nash equilibrium $s$ such that for all $i, p_{i}(s)>0$. By Theorem $8 s$ is of the form $\bar{t}$ for some product $t$. Then consider the following strategy profile:

$$
s:=\left(t, \ldots, t, t_{0}\right) \text {. }
$$


First schedule node 1 that has a better response, namely $t_{0}$. Next, schedule node $n$ for which $t$ is a best response. After these two steps the strategy profile becomes a rotation of $s$ by 1 . Iterating this selection procedure we obtain an infinite best response improvement path.

Next, suppose that the game $\mathcal{G}(\mathcal{S})$ has two determined Nash equilibria. By Theorem 8 they are of the form $\overline{t_{1}}$ and $\overline{t_{2}}$ for some products $t_{1}$ and $t_{2}$. Then consider the following strategy profile:

$$
s:=\left(t_{1}, \ldots, t_{1}, t_{2}\right) .
$$

First schedule node 1 for which $t_{2}$ is a best response. Next, schedule node $n$ for which $t_{1}$ is a best response. After these two steps the strategy profile becomes a rotation of $s$ by 1 . Iterating this selection procedure we obtain an infinite best response improvement path.

This theorem leads to a direct proof of the claimed result.

Proof of Theorem 18.

Thanks to the above theorem, one can check whether $\mathcal{G}(\mathcal{S})$ has the FBRP as follows. First apply the procedure VerifyNashCycle $(\mathcal{S})$ defined in the proof of Theorem 9, appropriately modified to check for the existence of a Nash equilibrium with strictly positive payoffs. If such an equilibrium does not exist, then we use a modified version of VerifyNashCycle $(\mathcal{S})$ to check whether $\mathcal{G}(\mathcal{S})$ has two determined Nash equilibria.

\subsection{Graphs with no source nodes}

As in Section 5 we conclude by considering social networks whose underlying graphs have no source nodes. The following counterpart of Theorem 16 holds.

Theorem 20. For a network $\mathcal{S}$ whose underlying graph has no source nodes, deciding whether the game $\mathcal{G}(\mathcal{S})$ has the FBRP is co-NP-hard.

Proof. The proof is a modification of the proof of Theorem 16. Given an instance of the PARTITION problem we use the following modification of the network given in Figure 6. We 'twin' each node $i \in\{1, \ldots, n\}$ with a new node $i^{\prime}$, also with the product set $\left\{t_{1}, t_{2}\right\}$, by adding the edges $\left(i, i^{\prime}\right)$ and $\left(i^{\prime}, i\right)$. We choose the weights $w_{i i^{\prime}}, w_{i^{\prime} i}$, where $i \in\{1, \ldots, n\}$ and the thresholds so that when $i$ and $i^{\prime}$ adopt a common product, their payoffs are positive.

Suppose that a solution to the considered instance of the PARTITION problem exists. Then we extend the joint strategy considered in the proof of Theorem 16 by additionally assigning $t_{1}$ to each node $i^{\prime}$ such that $i \in S$ and $t_{2}$ to each node $i^{\prime}$ such that $i \in\{1, \ldots, n\} \backslash S$. Then, as before, there is no finite best response improvement path starting in this joint strategy, so $\mathcal{G}(\mathcal{S})$ does not have the FBRP.

Suppose that an infinite best response improvement path $\xi$ exists in $\mathcal{G}(\mathcal{S})$. By Theorem 19(i) each network consisting of just the twined nodes $\left(1,1^{\prime}\right), \ldots,\left(n, n^{\prime}\right)$ has the FBRP. Therefore there exists a joint strategy $s^{k}$ in $\xi$ such that in the 
(infinite) suffix of $\xi$ starting at $s^{k}$ (call it $\xi_{k}$ ), none of the following nodes are scheduled: $1,1^{\prime}, \ldots, n, n^{\prime}$. The payoff for node $c$ in $\xi_{k}$ is at most 0 . Hence $t_{0}$ is always a best response for it. If $c$ chooses $t_{0}$ in $\xi$ then clearly $\xi$ is finite which contradicts the assumption. Also, if there exists a $s^{m}$ in $\xi_{k}$ such that $c$ never changes its strategy in the suffix of $\xi_{k}$ starting at $s_{m}$, then again $\xi$ is finite. This means that in $\xi_{k}$ the node $c$ alternates between the strategies $t_{1}$ and $t_{2}$. Hence in $\xi_{k}$ strategies $t_{1}$ and $t_{2}$ are also infinitely often selected by the nodes $a$ and $b$. Consequently, the payoff for the node $a$ at the moment it switches to $t_{1}$ has to be $\geq 0$ and the payoff for the node $b$ at the moment it switches to $t_{2}$ has to be $\geq 0$. This means that $\sum_{i \in\{1, \ldots, n\} \mid s_{i}=t_{1}} w_{i a} \geq \frac{1}{4}$ and $\sum_{i \in\{1, \ldots, n\} \mid s_{i}=t_{2}} w_{i b} \geq \frac{1}{4}$. But $\sum_{i=1}^{n} a_{i}=\frac{1}{2}$ and for $i \in\{1, \ldots, n\}, w_{i a}=w_{i b}=a_{i}$, and $s_{i} \in\left\{t_{1}, t_{2}\right\}$. So both above inequalities are in fact equalities. Consequently there exists a solution to the considered instance of the PARTITION problem.

\section{Finite improvement property: general case}

In the previous section we noted that some social network games do not have the FBRP. A fortiori, such games do not have the FIP either. We now analyze the complexity of determining whether a game has the FIP. We start with the following analogue of Theorem 16, though the proof is more complex.

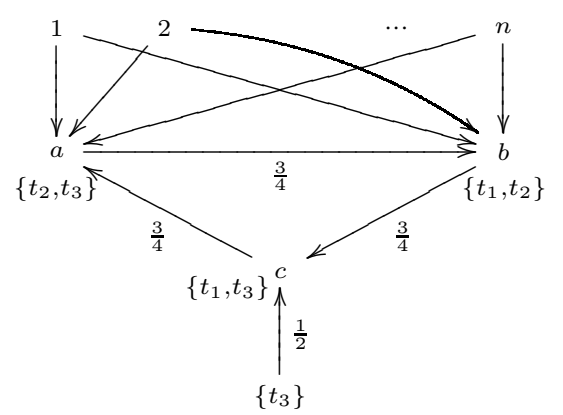

Figure 10: A network related to the FIP

Theorem 21. Deciding whether for a network $\mathcal{S}$ the game $\mathcal{G}(\mathcal{S})$ has the FIP is co-NP-hard.

Proof. We prove that the complement of the problem is NP-hard. We use again an instance of the PARTITION problem in the form of $n$ positive rational numbers $\left(a_{1}, \ldots, a_{n}\right)$, appropriately normalised, so that (this time) $\sum_{i=1}^{n} a_{i}=\frac{1}{2}$, and the network given in Figure 10. For each node $i \in\{1, \ldots, n\}$ we set the product set to be $\left\{t_{1}, t_{2}\right\}$ and $w_{i a}=w_{i b}=a_{i}$. The weights of the other edges are shown in the figure.

Since for all $i \in\{1, \ldots, n\}, a_{i}$ is rational, it has the form $a_{i}=\frac{l_{i}}{r_{i}}$. Let $\tau=\frac{1}{4 \cdot r_{1} \cdot \ldots \cdot r_{n}}$. The following property holds. 
Property 1. Given an instance $\left(a_{1}, \ldots, a_{n}\right)$ of the PARTITION problem and $\tau$ defined as above, for all $S \subseteq\{1, \ldots, n\}$

(i) if $\sum_{i \in S} a_{i}<\frac{1}{4}$, then $\sum_{i \in S} a_{i} \leq \frac{1}{4}-\tau$,

(ii) if $\sum_{i \in S} a_{i}>\frac{1}{4}$, then $\sum_{i \in S} a_{i} \geq \frac{1}{4}+\tau$.

Proof. By definition, each $a_{i}$ and $\frac{1}{4}$ is a multiple of $\tau$. Thus $\sum_{i \in S} a_{i}=x \cdot \tau$ and $\frac{1}{4}=y \cdot \tau$ where $x$ and $y$ are integers.

(i) If $x \cdot \tau<y \cdot \tau$, then $x \cdot \tau \leq(y-1) \cdot \tau$. Therefore $\sum_{i \in S} a_{i} \leq \frac{1}{4}-\tau$.

The proof of (ii) is analogous.

Note that given $\left(a_{1}, \ldots, a_{n}\right), \tau$ can be defined in polynomial time. Let the thresholds be defined as follows: $\theta\left(a, t_{2}\right)=\frac{1}{2}, \theta\left(a, t_{3}\right)=\frac{1}{4}+\tau, \theta\left(b, t_{1}\right)=\frac{1}{2}$, $\theta\left(b, t_{2}\right)=\frac{1}{2}+\tau$ and for node $c, \theta\left(c, t_{1}\right)=\theta\left(c, t_{3}\right)=\frac{1}{4}$.

Suppose now that a solution to the considered instance of the PARTITION problem exists. That is, for some set $S \subseteq\{1, \ldots, n\}$ we have $\sum_{i \in S} a_{i}=$ $\sum_{i \notin S} a_{i}=\frac{1}{4}$. Consider the game $\mathcal{G}(\mathcal{S})$. Assume now that strategy $t_{1}$ is selected by each node $i \in S$, strategy $t_{2}$ by each node $i \in\{1, \ldots, n\} \backslash S$ and that the node labelled by $\left\{t_{3}\right\}$ selected strategy $t_{3}$. Identify each completion of this selection to a joint strategy with the triple of strategies selected by the nodes $a, b$ and $c$. Then it is easy to check that Figure 11(a) exhibits an infinite improvement path in $\mathcal{G}(\mathcal{S})$; for convenience we list the corresponding payoffs in Figure 11(b).

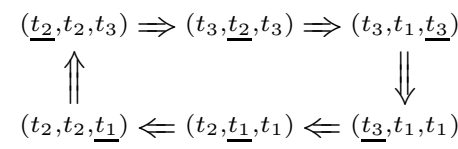

(a)

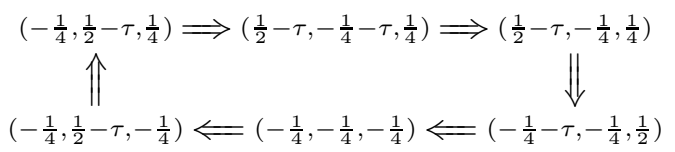

(b)

Figure 11: An infinite improvement path and the corresponding payoffs

Conversely, we show that if there is no solution to the considered instance of the PARTITION problem, then the game $\mathcal{G}(\mathcal{S})$ has the FIP. Consider an improvement path $\xi$. Let $k \geq 0$ be the first index (of a joint strategy) in $\xi$ such that in the suffix of $\xi$ starting at $k$, none of the source nodes is anymore scheduled. Let $[n]$ denote the set $\{1,2, \ldots, n\}, s^{k}$ the $k$-th element of $\xi, \operatorname{Val}\left(t_{1}\right)=$ $\sum_{i \in[n] \mid s_{i}^{k}=t_{1}} a_{i}$ and $\operatorname{Val}\left(t_{2}\right)=\sum_{i \in[n] \mid s_{i}^{k}=t_{2}} a_{i}$. There are two cases.

Case 1: $\operatorname{Val}\left(t_{2}\right)<\frac{1}{4}$. By Property 1, it follows that $\operatorname{Val}\left(t_{2}\right) \leq \frac{1}{4}-\tau$. We argue that $t_{2}$ is never a better response for node $a$. Suppose $s_{a}^{k}=t_{3}$. We have the following two possibilities:

- $s_{c}^{k}=t_{3}$; then $p_{a}\left(s^{k}\right)=\frac{1}{2}-\tau$, so $t_{3}$ is node $a$ 's best response,

- $s_{c}^{k} \neq t_{3}$; then $p_{a}\left(s^{k}\right)=-\frac{1}{4}-\tau$. If node $a$ switches to $t_{2}$, then the payoff is $p_{a}\left(s_{-a}^{k}, t_{2}\right) \leq\left(\frac{1}{4}-\tau\right)-\frac{1}{2}=-\frac{1}{4}-\tau$. Thus $t_{2}$ is not a better response for $a$. 
Similarly, it is easy to check that if $s_{a}^{k}=t_{0}$, then $t_{2}$ is not a better response for node $a$. If node $a$ never changes its strategy in $\xi$ then clearly $\xi$ is finite. If it does, then let $k_{a}>k$ be the first index in $\xi$ such that node $a$ changes its strategy (i.e., $s_{a}^{k} \neq s_{a}^{k_{a}}$ ). Since $t_{2}$ is never a better response for $a$, in the suffix of $\xi$ (call it $\xi_{a}$ ) starting at $k_{a}$, node $a$ never chooses $t_{2}$. In $\xi_{a}$ the better response of node $b$ can be $t_{1}, t_{2}$ or $t_{0}$. However, since node $a$ never chooses $t_{2}, b$ 's payoff in $\xi_{a}$ depends only on the choice made by the source nodes (which never changes in $\xi_{a}$ ). Therefore, there exists an index $k_{b}$ in $\xi$, where $k<k_{a}<k_{b}$, such that in the suffix of $\xi$ starting at $k_{b}$ (call it $\xi_{b}$ ), node $b$ never changes its strategy. The payoff of node $c$ depends on the choice made by nodes $b$ and the source node marked $\left\{t_{3}\right\}$. Since in $\xi_{b}$ node $b$ never changes its strategy, there is a suffix of $\xi_{b}$ in which node $c$ never changes its strategy. Therefore $\xi_{b}$, and hence $\xi$ is finite.

Case 2: $\operatorname{Val}\left(t_{2}\right)>\frac{1}{4}$. By Property 1, it follows that $\operatorname{Val}\left(t_{2}\right) \geq \frac{1}{4}+\tau$. We argue that $t_{1}$ is never a better response for node $b$. Suppose $s_{b}^{k}=t_{2}$. We have the following two possibilities:

- $s_{a}^{k}=t_{2}$; then $p_{b}\left(s^{k}\right) \geq \frac{1}{2}$, so $t_{2}$ is node $b$ 's best response,

- if $s_{a}^{k} \neq t_{2}$, then $p_{b}\left(s^{k}\right) \geq\left(\frac{1}{4}+\tau\right)-\frac{1}{2}-\tau=-\frac{1}{4}$. If node $b$ switches to $t_{1}$, then since $\operatorname{Val}\left(t_{1}\right)<\frac{1}{4}$, the payoff is $p_{b}\left(s_{-b}^{k}, t_{1}\right)<\frac{1}{4}-\frac{1}{2}=-\frac{1}{4}$. Thus $t_{1}$ is not a better response for $b$.

Similarly, it is easy to check that if $s_{b}^{k}=t_{0}$ then $t_{1}$ is not a better response for node $b$. This implies that there exists an index $k_{b}>k$ in $\xi$ such that for the suffix of $\xi$ (call it $\xi_{b}$ ) starting at $k_{b}$, node $b$ never chooses $t_{1}$. In $\xi_{b}$, since node $b$ never chooses $t_{1}$, the better response of node $c$ can be either $t_{3}$ or $t_{0}$. Thus $c$ 's payoff in $\xi_{b}$ depends only on the choice made by the source node labelled by $\left\{t_{3}\right\}$. Therefore, there exists an index $k_{c}$ in $\xi$, where $k<k_{b}<k_{c}$, such that in the suffix of $\xi$ starting at $k_{c}$ (call it $\xi_{c}$ ), node $c$ is never scheduled. It follows that $\xi_{c}$ and hence $\xi$ is finite.

Since the choice of the improvement path was arbitrary, it follows that $\mathcal{G}(\mathcal{S})$ has the FIP.

\section{Finite improvement property: special cases}

In this section we clarify whether the special classes of social network games have the FIP and if not, what the complexity of the problem is.

First, we would like to mention the following question to which we did not succeed to find an answer.

Open problem: Consider a network $\mathcal{S}$ whose underlying graph is a simple cycle. What is the complexity of determining whether $\mathcal{G}(\mathcal{S})$ has the FIP?

In what follows we make use of the following simple observation.

Note 22. Consider a game $\mathcal{G}(\mathcal{S})$. If a node $i$ is infinitely often selected in an improvement path, then so is a node $j \in N(i)$. 


\subsection{Graphs with special strongly connected components}

We begin with the strategic games associated with the networks whose underlying graph is a DAG. Then the following positive result holds.

Theorem 23. Consider a network $\mathcal{S}$ whose underlying graph is a DAG. Then the game $\mathcal{G}(\mathcal{S})$ has the FIP.

Proof. Suppose not. Then by repeatedly using Note 22 we obtain an infinite path in the underlying graph. This yields a contradiction.

We now generalize this result to a larger class of directed graphs. First we consider the case of two player games.

Theorem 24. Every two player social network game has the FIP.

Proof. By Theorem 23 we can assume that the underlying graph is a cycle, say $1 \rightarrow 2 \rightarrow 1$. Consider an improvement path $\rho$. By removing, if necessary, some steps we can assume that the players alternate their moves in $\rho$.

In what follows given an element of $\rho$ (that is not the last one) we underline the strategy of the player who moves, i.e., selects a better response. We call each element of $\rho$ of the type $(\underline{t}, t)$ or $(t, \underline{t})$ a match and use $\Rightarrow$ to denote the transition between two consecutive joint strategies in $\rho$. Further, we shorten the statement "each time player $i$ switches his strategy his payoff strictly increases and it never decreases when his opponent switches strategy" to "player $i$ 's payoff steadily goes up".

Consider now two successive matches in $\rho$, based respectively on the strategies $t$ and $t_{1}$. The corresponding segment of $\rho$ is one of the following four types. Type 1. $(\underline{t}, t) \Rightarrow^{*}\left(\underline{t_{1}}, t_{1}\right)$.

The fragment of $\rho$ that starts at $(\underline{t}, t)$ and finishes at $\left(\underline{t_{1}}, t_{1}\right)$ has the following form:

$$
(\underline{t}, t) \Rightarrow\left(t_{2}, \underline{t}\right) \Rightarrow^{*}\left(t_{1}, \underline{t_{3}}\right) \Rightarrow\left(\underline{t_{1}}, t_{1}\right) .
$$

Note that player 1's payoff can drop in a segment of $\rho$ only if this segment contains a transition of the form $\left(t^{\prime}, \underline{t^{\prime}}\right) \Rightarrow\left(\underline{t^{\prime}}, t_{1}\right)$. So in the considered segment player 1 's payoff steadily goes up. Additionally, in the step $\left(t_{1}, \underline{t_{3}}\right) \Rightarrow\left(\underline{t_{1}}, t_{1}\right)$ his payoff increases by $w_{21}$.

In turn, in the step $(\underline{t}, t) \Rightarrow\left(t_{2}, \underline{t}\right)$ player 2 's payoff decreases by $w_{12}$ and in the remaining steps his payoff steadily goes up. So $p_{1}(\bar{t})+w_{21}<p_{1}\left(\overline{t_{1}}\right)$ and $p_{2}(\bar{t})-w_{12}<p_{2}\left(\overline{t_{1}}\right)$.

Type 2. $(\underline{t}, t) \Rightarrow^{*}\left(t_{1}, \underline{t_{1}}\right)$.

For the analogous reason as above player 1's payoff steadily goes up. In turn, in the first step of $(\underline{t}, t) \Rightarrow^{*}\left(t_{1}, \underline{t_{1}}\right)$ the payoff of player 2 decreases by $w_{12}$, while in the last step (in which player 1 moves) his payoff increases by $w_{12}$. So these two payoff changes cancel each other. Additionally, in the remaining steps player 2's payoff steadily goes up. So $p_{1}(\bar{t})<p_{1}\left(\overline{t_{1}}\right)$ and $p_{2}(\bar{t})<p_{2}\left(\overline{t_{1}}\right)$.

Type 3. $(t, \underline{t}) \Rightarrow^{*}\left(\underline{t_{1}}, t_{1}\right)$.

This type is symmetric to Type 2 , so $p_{1}(\bar{t})<p_{1}\left(\overline{t_{1}}\right)$ and $p_{2}(\bar{t})<p_{2}\left(\overline{t_{1}}\right)$. 
Type 4. $(t, \underline{t}) \Rightarrow^{*}\left(t_{1}, \underline{t_{1}}\right)$.

This type is symmetric to Type 1 , so $p_{1}(\bar{t})-w_{21}<p_{1}\left(\overline{t_{1}}\right)$ and $p_{2}(\bar{t})+w_{12}<$ $p_{2}\left(\overline{t_{1}}\right)$.

We summarize in Table 1 the changes in the payoffs $p_{1}$ and $p_{2}$ between the considered two matches.

\begin{tabular}{|l|l|l|}
\hline Type & $p_{1}$ & $p_{2}$ \\
\hline \hline 1 & $\begin{array}{l}\text { increases } \\
\text { by }>w_{21}\end{array}$ & $\begin{array}{l}\text { decreases } \\
\text { by }<w_{12}\end{array}$ \\
\hline 2,3 & increases & increases \\
\hline 4 & $\begin{array}{l}\text { decreases } \\
\text { by }<w_{21}\end{array}$ & $\begin{array}{l}\text { increases } \\
\text { by }>w_{12}\end{array}$ \\
\hline
\end{tabular}

Table 1: Changes in $p_{1}$ and $p_{2}$

Consider now a match $(\underline{t}, t)$ in $\rho$ and a match $\left(\underline{t_{1}}, t_{1}\right)$ that appears later in $\rho$. Let $T_{i}$ denote the number of internal segments of type $i$ that occur in the fragment of $\rho$ that starts with $(\underline{t}, t)$ and ends with $\left(\underline{t_{1}}, t_{1}\right)$.

Case 1. $T_{1} \geq T_{4}$.

Then Table 1 shows that the aggregate increase in $p_{1}$ in segments of type 1 exceeds the aggregate decrease in segments of type 4 . So $p_{1}(\bar{t})<p_{1}\left(\overline{t_{1}}\right)$.

Case 2. $T_{1}<T_{4}$.

Then analogously Table 1 shows that $p_{2}(\bar{t})<p_{2}\left(\overline{t_{1}}\right)$.

We conclude that $t \neq t_{1}$. By symmetry the same conclusion holds if the considered matches are of the form $(t, \underline{t})$ and $\left(t_{1}, \underline{t_{1}}\right)$. This proves that each match occurs in $\rho$ at most once. So in some suffix $\eta$ of $\rho$ no match occurs. But each step in $\eta$ increases the social welfare, so $\eta$ is finite, and consequently $\rho$ is.

In social network games the players share at least one strategy, $t_{0}$, that ensures each of them the zero payoff. Also, the weights and thresholds are drawn from specific intervals. However, these properties are not used in the above proof. As a result the above proof shows that each of the following two player games has the FIP.

- The set of strategies of player $i$ is a finite set $S_{i}$,

- the payoff function is defined by $p_{i}(s):=f_{i}\left(s_{i}\right)+a_{i}\left(s_{i}=s_{-i}\right)$, where $f_{i}: S_{i} \rightarrow \mathbb{R}, a_{i}>0$ and $\left(s_{i}=s_{-i}\right)$ is defined by

$$
\left(s_{i}=s_{-i}\right):= \begin{cases}1 & \text { if } s_{i}=s_{-i} \\ 0 & \text { otherwise }\end{cases}
$$


Intuitively, $a_{i}$ can be viewed as a bonus for player $i$ for coordinating with his opponent.

It is worthwhile to notice that if we allowed that the weights depend on the product and modified the definition of the payoff function to

$$
\sum_{j \in \mathcal{N}_{i}^{t}(s)} w_{j i}(t)-\theta(i, t)
$$

then the above result would not hold.

Example 25. Suppose that the product sets of player 1 and player 2 are $\left\{t_{1}, t_{2}, t_{3}\right\}$ and $\left\{t_{1}, t_{3}, t_{4}\right\}$ respectively. Define the threshold functions as follows:

$$
\begin{aligned}
& \theta\left(1, t_{1}\right)=0.9, \theta\left(1, t_{2}\right)=0.8, \theta\left(1, t_{3}\right)=1, \\
& \theta\left(2, t_{1}\right)=1, \theta\left(2, t_{3}\right)=0.9, \theta\left(2, t_{4}\right)=0.8,
\end{aligned}
$$

and suppose the parametrized weight functions are such that

$$
w_{21}\left(t_{1}\right)=0, w_{21}\left(t_{3}\right)=0.3, w_{12}\left(t_{1}\right)=0.3, w_{12}\left(t_{3}\right)=0 .
$$

Then it is easy to check that the iteration of the following sequence forms an infinite improvement path:

$$
\left(\underline{t_{1}}, t_{1}\right) \Rightarrow\left(t_{2}, \underline{t_{1}}\right) \Rightarrow\left(\underline{t_{2}}, t_{3}\right) \Rightarrow\left(t_{3}, \underline{t_{3}}\right) \Rightarrow\left(\underline{t_{3}}, t_{4}\right) \Rightarrow\left(t_{1}, \underline{t_{4}}\right) \Rightarrow\left(\underline{t_{1}}, t_{1}\right) .
$$

We can now draw a conclusion about a larger class of social network games.

Theorem 26. Consider a network $\mathcal{S}$ such that each strongly connected component of the underlying graph is a cycle of length 2. Then the game $\mathcal{G}(\mathcal{S})$ has the FIP.

Proof. Suppose $\mathcal{S}=(G, \mathcal{P}, P, \theta)$. Consider the condensation of $G$, i.e., the DAG $G^{\prime}$ resulting from contracting each cycle of $G$ to a single node. We enumerate the nodes of $G$ using the function $\operatorname{rank}()$ (introduced in Subsection 5.1) such that if $\operatorname{rank}(i)<\operatorname{rank}(j)$, then there is no path in $G$ from $j$ to $i$, and subsequently modify it to an enumeration $\operatorname{rank}^{\prime}()$ of the nodes of $G^{\prime}$, by replacing each contraction of a cycle by its two nodes.

Suppose now that an infinite improvement path $\rho$ in $\mathcal{G}(\mathcal{S})$ exists. Choose the first node $i$ in the enumeration $\operatorname{rank}^{\prime}()$ that is infinitely often selected in $\rho$. By Note $22 i$ lies on a cycle, say $i \rightarrow i^{\prime} \rightarrow i$, in $G$. Consider a suffix of $\rho$ in which the nodes that precede $i$ in $\operatorname{rank}^{\prime}()$ do not appear anymore. In particular, by the choice of $i$, the neighbours of $i$ or $i^{\prime}$ precede $i$ in $\operatorname{rank}^{\prime}()$, so they do not appear in this suffix either. Delete from each element of this suffix the strategies of the nodes that differ from $i$ and $i^{\prime}$. We obtain in this way an infinite improvement path in the two player game $G^{\prime}$ associated with the weighted directed graph related to the cycle $i \rightarrow i^{\prime} \rightarrow i$, which contradicts Theorem 24 .

There is a small subtlety in the last step that we should clarify. The payoff functions in the game $G^{\prime}$ need to take into account the weights of the edges from 
all the neighbours of $i$ and $i^{\prime}$ and the final strategies chosen by these neighbours. For instance, in the case of the directed graph from Figure 12 the game $G^{\prime}$ has the players $i$ and $i^{\prime}$ but the weights of the edges $k \rightarrow i$ and $k \rightarrow i^{\prime}$ and the final strategy of node $k$ need to be taken into account in the computation of the payoff functions for, respectively, nodes $i$ and $i^{\prime}$.

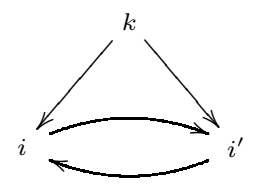

Figure 12: A directed graph

So the game $G^{\prime}$ is not exactly the social network game associated with the weighted directed graph related to $i \rightarrow i^{\prime} \rightarrow i$. However, the observation stated after the proof of Theorem 24 allows us to conclude that $G^{\prime}$ does have the FIP.

\subsection{Graphs with no source nodes}

We conclude with the following analogue of Theorem 20.

Theorem 27. For a network $\mathcal{S}$ whose underlying graph has no source nodes, deciding whether the game $\mathcal{G}(\mathcal{S})$ has the FIP is co-NP-hard.

Proof. The proof is a modification of Theorem 21. Given an instance of the PARTITION problem we use the following modification of the network given in Figure 10. We 'twin' each node $i \in\{1, \ldots, n\}$ with a new node $i^{\prime}$, also with the product set $\left\{t_{1}, t_{2}\right\}$, by adding the edges $\left(i, i^{\prime}\right)$ and $\left(i^{\prime}, i\right)$. We also 'twin' the node marked $\left\{t_{3}\right\}$, call it $d$, with a new node $d^{\prime}$ also with the product set $\left\{t_{3}\right\}$, by adding the edges $\left(d, d^{\prime}\right)$ and $\left(d^{\prime}, d\right)$. Next, we choose the weights $w_{i i^{\prime}}, w_{i^{\prime} i}$, where $i \in\{1, \ldots, n\}$, and $w_{d d^{\prime}}$ and $w_{d^{\prime} d}$ and the corresponding thresholds so that when $i$ and $i^{\prime}$ or $d$ and $d^{\prime}$ adopt a common product, their payoffs are positive.

Suppose that a solution to the considered instance of the PARTITION problem exists. Then we extend the joint strategy considered in the proof of Theorem 21 by additionally assigning $t_{1}$ to each node $i^{\prime}$ such that $i \in S, t_{2}$ to each node $i^{\prime}$ such that $i \in\{1, \ldots, n\} \backslash S$ and $t_{3}$ to nodes $d$ and $d^{\prime}$. Then, as before, there is no finite improvement path starting in this joint strategy, so $\mathcal{G}(\mathcal{S})$ does not have the FIP.

Suppose that no solution to the considered instance of the PARTITION problem exists. We show that then the game has the FIP. Consider an improvement path $\xi$. By Theorem 24, each network consisting of just the twined nodes $\left(1,1^{\prime}\right), \ldots,\left(n, n^{\prime}\right)$ and $\left(d, d^{\prime}\right)$ has the FIP. Therefore, there exists a joint strategy $s^{k}$ in $\xi$ such that in the suffix of $\xi$ starting at $s^{k}$, none of the following nodes are scheduled: $1,1^{\prime}, \ldots, n, n^{\prime}, d, d^{\prime}$. Again, we have the two cases as analysed in Theorem 21. When $\operatorname{Val}\left(t_{2}\right)<\frac{1}{4}$, using the same argument as in Theorem 21 we conclude that the improvement path $\xi$ is finite. In the case when $\operatorname{Val}\left(t_{2}\right)>\frac{1}{4}$ 
the argument in Theorem 21 shows that there exists an index $k_{b}>k$ in $\xi$ such that for the suffix of $\xi$ starting at $k_{b}$ (call it $\xi_{b}$ ), node $b$ never chooses $t_{1}$. If $d$ and $d^{\prime}$ choose $t_{0}$ in $s^{k}$, since $b$ never chooses $t_{1}$ in $\xi_{b}$, the better response of $c$ in $\xi_{b}$ is $t_{0}$. From this it follows that $\xi_{b}$ and hence $\xi$ is finite. If $d$ and $d^{\prime}$ choose $t_{3}$ in $s^{k}$, then it follows by the argument given in Theorem 21 that $\xi$ is finite.

\section{The uniform FIP property: general case}

Let us reconsider the games associated with the networks from Figures 8(a) and 9(a). Since these games do not have the FBRP, by definition, they do not have the FIP either. In spite of this fact, for any initial joint strategy there exists a finite improvement path. Indeed, it suffices to schedule the players in the clockwise manner, that is to choose each time the first player who did not select a best response. This is an instance of a more general result proved in the next section.

By a scheduler we mean a function $f$ that given a joint strategy $s$ that is not a Nash equilibrium selects a player who did not select a best response in $s$. An improvement path $\xi=s^{1}, s^{2}, \ldots$ respects a scheduler $f$ if for all $k$ smaller than the length of $\xi, s^{k+1}=\left(s_{i}^{\prime}, s_{-i}^{k}\right)$, where $f\left(s^{k}\right)=i$. We say that a strategic game has the uniform $\boldsymbol{F I P}$ if there exists a scheduler $f$ such that all improvement paths $\rho$ which respect $f$ are finite.

In this and the next section we investigate the complexity of determining whether a social network game has the uniform FIP. We begin by establishing the following counterpart of Theorem 21.

Theorem 28. Deciding whether for a network $\mathcal{S}$ the game $\mathcal{G}(\mathcal{S})$ has the uniform FIP is co-NP-hard.

Proof. We prove that the complement of the problem is NP-hard. As before we use an instance of the PARTITION problem in the form of $n$ positive rational numbers $\left(a_{1}, \ldots, a_{n}\right)$ such that $\sum_{i=1}^{n} a_{i}=1$. To construct the appropriate network $\mathcal{S}$, as in the proof of Theorem 1, we employ the networks given in Figures 1 and 2. However, we use different product sets in the second network and combine these two networks differently.

For each node $i \in\{1, \ldots, n\}$ we set $P(i)=\left\{t_{1}, t_{2}\right\}$. Further we set $P(a)=$ $\left\{t_{1}\right\}$ and $P(b)=\left\{t_{2}\right\}$. As before we set $w_{i a}=w_{i b}=a_{i}$ and we assume that the threshold of the nodes $a$ and $b$ is constant and equals $\frac{1}{2}$. We now identify the nodes $a$ and $b$ of the network from Figure 2 respectively with the nodes marked by $\left\{t_{1}\right\}$ and $\left\{t_{2}\right\}$ in the network in Figure 1. The resulting network is given in Figure 13.

Suppose now that a solution to the considered instance of the PARTITION problem exists, that is for some set $S \subseteq\{1, \ldots, n\}$ we have $\sum_{i \in S} a_{i}=\sum_{i \notin S} a_{i}=$ $\frac{1}{2}$. Consider the game $\mathcal{G}(\mathcal{S})$. Take the joint strategy $s$ formed by the following strategies:

- $t_{1}$ assigned to each node $i \in S$ and the nodes $a$ and $c$, 


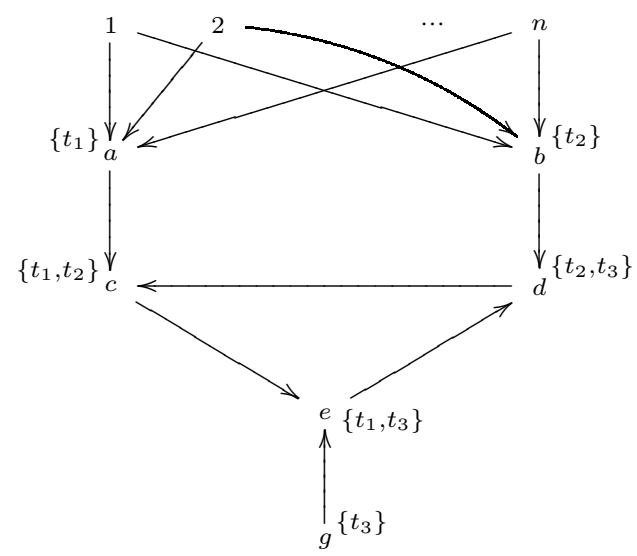

Figure 13: A network related to the uniform FIP

- $t_{2}$ assigned to each node $i \in\{1, \ldots, n\} \backslash S$ and the nodes $b$ and $d$,

- $t_{3}$ assigned to the nodes $e$ and $g$.

Any improvement path that starts in this joint strategy will not change the strategies assigned to the nodes $a, b$ and $g$. So if such an improvement path terminates, it produces a Nash equilibrium in the game associated with the network given in Figure 1 of Example 2. But we showed there that this game does not have a Nash equilibrium. Consequently, there is no finite improvement path in the game $\mathcal{G}(\mathcal{S})$ that starts in the above joint strategy and a fortiori $\mathcal{G}(\mathcal{S})$ does not have the uniform FIP.

Suppose now that no solution to the considered instance of the PARTITION problem exists. We show that then the game $\mathcal{G}(\mathcal{S})$ has the uniform FIP. To this end we order the nodes of $\mathcal{S}$ as follows (note the positions of the nodes $c, d$ and $e)$ :

$$
1,2, \ldots, n, a, b, g, c, e, d .
$$

Consider the scheduler $f$ that given a joint strategy selects the first node in the above list that did not select a best response. (An aside: a node can be selected more than once - such a possibility is discussed in the second case below.)

Take an improvement path that respects this scheduler. After at most $n$ steps the nodes $1,2, \ldots, n$ all selected a product $t_{1}$ or $t_{2}$. Suppose first that $\sum_{i \in\{1, \ldots, n\} \mid s_{i}=t_{1}} w_{i a}>\frac{1}{2}$.

In the considered improvement path eventually the following selections have been made by the nodes $a, b, g, c$ and $e$ :

$$
a: t_{1}, b: t_{0}, g: t_{3}, c: t_{1}, e: t_{1} \text {. }
$$

Either the node $d$ initially selected $t_{0}$ or it is eventually scheduled and then it switches to $t_{0}$. At this moment a Nash equilibrium is reached, that is, the considered improvement path is finite. 
Suppose next that $\sum_{i \in\{1, \ldots, n\} \mid s_{i}=t_{2}} w_{i b}>\frac{1}{2}$. Then after a number of steps the following selections have been made by the nodes $a, b$ and $g$ :

$$
a: t_{0}, b: t_{2}, g: t_{3}
$$

Either the node $c$ initially selected $t_{0}$ or $t_{2}$, or it is eventually scheduled and then it switches to $t_{0}$ or $t_{2}$. Further, either the node $e$ initially selected $t_{3}$ or it is eventually scheduled and then it switches to $t_{3}$. Finally, if the node $d$ is eventually scheduled, then it switches to $t_{3}$, as well. If now the node $c$ is scheduled again, then it switches to $t_{0}$. At this moment a Nash equilibrium is reached and the considered improvement path terminates.

\section{The uniform FIP: special cases}

As in the case of the Nash equilibria and the FIP we now consider the uniform FIP for the special cases of social network games.

\subsection{Simple cycles}

In the case when the underlying graph is a simple cycle the following positive result holds.

Theorem 29. Let $\mathcal{S}$ be a network such that the underlying graph is a simple cycle. Then the game $\mathcal{G}(\mathcal{S})$ has the uniform FIP.

Proof. We use the scheduler $f$ that given a joint strategy $s$ chooses the smallest index $i$ such that $s_{i}$ is not a best response to $s_{-i}$. So this scheduler selects a player again if he did not switch to a best response. Therefore we can assume that each selected player immediately selects a best response.

Consider a joint strategy $s$ taken from a best response improvement path. Observe that for all $k$ if $s_{k} \in P(k)$ and $p_{k}(s) \geq 0$ (so in particular if $s_{k}$ is a best response to $s_{-k}$ ), then $s_{k}=s_{k \ominus 1}$. Consequently the following property holds for all $i>1$ :

$Z(i)$ : if $f(s)=i$ and $s_{i-1} \in P(i-1)$ then for all $j \in\{n, 1, \ldots, i-1\}, s_{j}=s_{i-1}$.

In words: if $i$ is the first player who did not choose a best response and player $i-1$ strategy is a product, then this product is the strategy of every earlier player and of player $n$.

Along each best response improvement path that respects $f$ the value of $f(s)$ strictly increases until the path terminates or at certain stage $f(s)=n$. In the latter case if $s_{n-1}=t_{0}$, then the unique best response for player $n$ is $t_{0}$. Otherwise $s_{n-1} \in P(n-1)$, so on the account of property $Z(n)$ all players' strategies equal the same product and the payoff of player $n$ is negative (since $f(s)=n)$. So the unique best response for player $n$ is $t_{0}$, as well.

This switch begins a new round with player 1 as the next scheduled player. Player 1 also switches to $t_{0}$ and from now on every consecutive player switches to $t_{0}$, as well. The resulting path terminates once player $n-2$ switches to $t_{0}$. 
Another scheduler $f$ that we could use is the one that given $s$ chooses the smallest index $i$ such that $p_{i}(s)<0$. Note that if $p_{i}(s)<0$, then $i$ does not play a best response in $s$. The argument is the same as for the above scheduler.

These two schedulers differ. Take for instance the network given in Figure 9 and the initial joint strategy $\left(t_{2}, t_{2}, t_{1}\right)$. The following improvement path terminating in the trivial Nash equilibrium respects the second scheduler:

$$
\left(\underline{t_{2}}, t_{2}, t_{1}\right) \Rightarrow\left(t_{0}, \underline{t_{2}}, t_{1}\right) \Rightarrow\left(t_{0}, t_{0}, \underline{t_{1}}\right) \Rightarrow\left(t_{0}, t_{0}, t_{0}\right) \text {. }
$$

However, using the first scheduler, node 1 is scheduled again in the joint strategy $\left(t_{0}, t_{2}, t_{1}\right)$ since $t_{0}$ is not its best response and similarly for node 2 . Thus the improvement path terminates in the Nash equilibrium $\left(t_{1}, t_{1}, t_{1}\right)$.

\subsection{Graphs with no source nodes}

Finally, we consider social network games in the case when the underlying graph has no source nodes. Then it is possible that for some initial joint strategy no improvement path starting at it terminates, so in particular the game does not have the uniform FIP. To see this consider the network given in Figure 14(a).

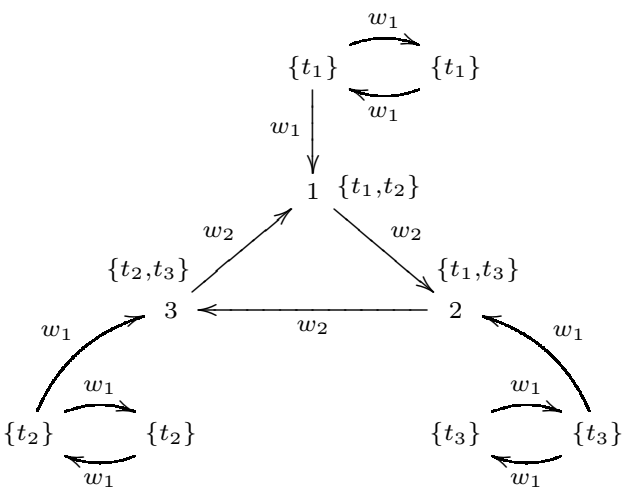

(a)

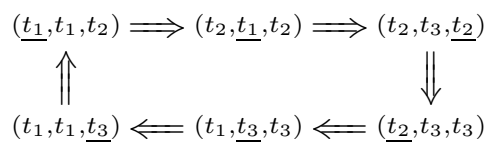

(b)

Figure 14: Another network with an infinite improvement path

We assume that each threshold is a constant $\theta$, where $\theta<w_{1}<w_{2}$. Consider the joint strategy $s$, in which the nodes marked by $\left\{t_{1}\right\},\left\{t_{2}\right\}$ and $\left\{t_{3}\right\}$ choose the unique product in their product sets and players 1,2 and 3 choose $t_{1}, t_{1}$ and $t_{2}$, respectively. For convenience, we denote $s$ by $\left(t_{1}, t_{1}, t_{2}\right)$. This joint strategy is not a Nash equilibrium since player 1 gains by deviating to $t_{2}$. There is a unique improvement path starting at $s$ and this path is infinite. It is shown in Figure 14(b). In each joint strategy, we underline the strategy that is not a best response.

By using a more complex network that builds upon the above one we now prove the following analogue of Theorem 28. 
Theorem 30. For a network $\mathcal{S}$ whose underlying graph has no source nodes, deciding whether the game $\mathcal{G}(\mathcal{S})$ has the uniform FIP is co-NP-hard.

Proof. The proof extends the proof of Theorem 28. Given an instance of the PARTITION problem we use the following modification of the network given in Figure 13. We 'twin' each node $i \in\{1, \ldots, n\}$ with a new node $i$ ', also with the product set $\left\{t_{1}, t_{2}\right\}$, by adding the edges $\left(i, i^{\prime}\right)$ and $\left(i^{\prime}, i\right)$. We also 'twin' the node $g$ with a new node $g^{\prime}$, also with the product set $\left\{t_{3}\right\}$, by adding the edges $\left(g, g^{\prime}\right)$ and $\left(g^{\prime}, g\right)$. Next, we choose the weights $w_{i i^{\prime}}, w_{i^{\prime} i}$, where $i \in\{1, \ldots, n\}$, and $w_{g g^{\prime}}$ and $w_{g^{\prime} g}$ and the corresponding thresholds so that when $i$ and $i^{\prime}$ or $g$ and $g^{\prime}$ adopt a common product, their payoff is positive.

Suppose that a solution to the considered instance of the PARTITION problem exists. Then we extend the joint strategy considered in the proof of Theorem 28 by additionally assigning $t_{1}$ to each node $i^{\prime}$ such that $i \in S, t_{2}$ to each node $i^{\prime}$ such that $i \in\{1, \ldots, n\} \backslash S$ and $t_{3}$ to the node $g^{\prime}$. Then, as before, there is no finite improvement path starting in this joint strategy, so $\mathcal{G}(\mathcal{S})$ does not have uniform FIP.

Suppose now that no solution to the considered instance of the PARTITION problem exists. We show that then the game $\mathcal{G}(\mathcal{S})$ has the uniform FIP. To this end we now use the following order of the nodes of $\mathcal{S}$ :

$$
1,1^{\prime}, 2,2^{\prime}, \ldots, n, n^{\prime}, g, g^{\prime}, a, b, c, e, d,
$$

and as before use the scheduler that 'follows' this ordering. (The reader may notice that the primed nodes will never be scheduled.)

When considering the possible joint strategies in $\mathcal{G}(\mathcal{S})$ we now need to consider new cases when after being scheduled some of the nodes $i \in\{1, \ldots, n\}$ or $g$ end up selecting the strategy $t_{0}$. In each case the reasoning is similar as in the proof of Theorem 28 and allows us to conclude that each improvement path terminates with a Nash equilibrium. We only mention the final selection of the strategies by the nodes $a, b, c, d, e$ and $g$ and leave checking the details to the reader.

Case 1. $\sum_{i \in\{1, \ldots, n\} \mid s_{i}=t_{1}} w_{i a}<\frac{1}{2}, \sum_{i \in\{1, \ldots, n\} \mid s_{i}=t_{2}} w_{i b}<\frac{1}{2}$ and $g$ selects $t_{3}$.

The final selection of the strategies by the nodes $a, b, c, d$ and $e$ is then

$$
a: t_{0}, b: t_{0}, c: t_{0}, d: t_{3}, e: t_{3} .
$$

Case 2. $\sum_{i \in\{1, \ldots, n\} \mid s_{i}=t_{1}} w_{i a}<\frac{1}{2}, \sum_{i \in\{1, \ldots, n\} \mid s_{i}=t_{2}} w_{i b}<\frac{1}{2}$ and $g$ selects $t_{0}$.

The final selection of the strategies by the nodes $a, b, c, d$ and $e$ is then

$$
a: t_{0}, b: t_{0}, c: t_{0}, d: t_{0}, e: t_{0} .
$$

Case 3. $\sum_{i \in\{1, \ldots, n\} \mid s_{i}=t_{1}} w_{i a} \geq \frac{1}{2}, \sum_{i \in\{1, \ldots, n\} \mid s_{i}=t_{2}} w_{i b}<\frac{1}{2}$ and $g$ selects $t_{3}$.

The final selection of the strategies by the nodes $a, b, c, d$ and $e$ is then

$$
a: t_{1}, b: t_{0}, c: t_{1}, d: t_{0}, e: t_{1} \text { or } a: t_{0}, b: t_{0}, c: t_{0}, d: t_{3}, e: t_{3},
$$

(where the latter can only arise when $\sum_{i \in\{1, \ldots, n\} \mid s_{i}=t_{1}} w_{i a}=\frac{1}{2}$ ). 
Case 4. $\sum_{i \in\{1, \ldots, n\} \mid s_{i}=t_{1}} w_{i a} \geq \frac{1}{2}, \sum_{i \in\{1, \ldots, n\} \mid s_{i}=t_{2}} w_{i b}<\frac{1}{2}$ and $g$ selects $t_{0}$.

The final selection of the strategies by the nodes $a, b, c, d$ and $e$ is then

$$
a: t_{1}, b: t_{0}, c: t_{1}, d: t_{0}, e: t_{1} \text { or } a: t_{0}, b: t_{0}, c: t_{0}, d: t_{0}, e: t_{0}
$$

(where the latter can only arise when $\sum_{i \in\{1, \ldots, n\} \mid s_{i}=t_{1}} w_{i a}=\frac{1}{2}$ ).

Case 5. $\sum_{i \in\{1, \ldots, n\} \mid s_{i}=t_{1}} w_{i a}<\frac{1}{2}, \sum_{i \in\{1, \ldots, n\} \mid s_{i}=t_{2}} w_{i b} \geq \frac{1}{2}$ and $g$ selects $t_{3}$.

The final selection of the strategies by the nodes $a, b, c, d$ and $e$ is then

$$
a: t_{0}, b: t_{2}, c: t_{0}, d: t_{3}, e: t_{3} \text { or } a: t_{0}, b: t_{0}, c: t_{0}, d: t_{3}, e: t_{3}
$$

(where the latter can only arise when $\sum_{i \in\{1, \ldots, n\} \mid s_{i}=t_{2}} w_{i b}=\frac{1}{2}$ ).

Case 6. $\sum_{i \in\{1, \ldots, n\} \mid s_{i}=t_{1}} w_{i a}<\frac{1}{2}, \sum_{i \in\{1, \ldots, n\} \mid s_{i}=t_{2}} w_{i b} \geq \frac{1}{2}$ and $g$ selects $t_{0}$.

The final selection of the strategies by the nodes $a, b, c, d$ and $e$ is then

$$
a: t_{0}, b: t_{2}, c: t_{2}, d: t_{2}, e: t_{0} \text { or } a: t_{0}, b: t_{0}, c: t_{0}, d: t_{0}, e: t_{0}
$$

(where the latter can only arise when $\sum_{i \in\{1, \ldots, n\} \mid s_{i}=t_{2}} w_{i b}=\frac{1}{2}$ ).

This proves that $\mathcal{G}(\mathcal{S})$ has the uniform FIP.

Analogously to the notion of a game having the uniform FIP one can introduce the notion of a game having the uniform $\boldsymbol{F B R P}$, by simply restricting attention to the best response improvement paths. It is easy to check that all the proofs given in this section show that the obtained results also hold when we replace the reference to the uniform FIP by that to the uniform FBRP. In fact, the latter property admits less improvement paths, so some proofs become simpler. We shall return to this matter in the last section.

\section{Weakly acyclic games}

Finally, we show that for the social network games the property of being weakly acyclic is weaker than that of having the uniform FIP.

Example 31. Consider the network $\mathcal{S}$ given in Figure 15(a). This is essentially the network in Figure 1 except for the addition of the new source node with the product set $\left\{t_{4}\right\}$ as a neighbour of node 1 and the new product $t_{4}$ included in the product sets of players 1,2 and 3 . We assume that each threshold is a constant $\theta$, where $\theta<w_{3}<w_{1}<w_{2}$.

We first show that the game $\mathcal{G}(\mathcal{S})$ does not have the uniform FIP. Assume that each source node selects its unique product. Identify each joint strategy that extends this selection with the selection of the strategies by the players 1 , 2 and 3 . Consider the infinite improvement path starting at the joint strategy $s=\left(t_{1}, t_{1}, t_{2}\right)$ shown in Figure 15(b). In each joint strategy in this improvement path, there is a unique player who is not playing his best response. Therefore, irrespective of the scheduler chosen, there is always a unique player who can be scheduled. It follows that $\mathcal{G}(\mathcal{S})$ does not have the uniform FIP. 


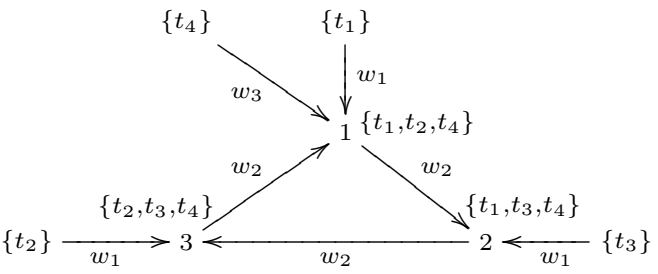

(a)

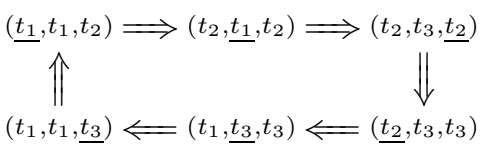

(b)

Figure 15: A weakly acyclic game that does not have the uniform FIP

On the other hand, this game is weakly acyclic. It suffices to consider joint strategies in which each source node selects its unique product. First note that any improvement path in which player 1 never adopts product $t_{2}$ is finite since he cannot switch from $t_{1}$ to $t_{4}$. We have the following cases, where as above, we only list the strategies of players 1,2 and 3 .

Case 1. $s=\left(t_{2}, x, y\right)$ where $y \neq t_{2}$. Then by definition $p_{1}(s)<0$. If $x \neq t_{4}$ then consider the improvement path $s=\left(\underline{t_{2}}, x, y\right) \Rightarrow\left(t_{4}, \underline{x}, y\right) \Rightarrow\left(t_{4}, t_{4}, \underline{y}\right) \Rightarrow$ $\left(t_{4}, t_{4}, t_{4}\right)$. So player 1 first switches to $t_{4}$ (which is a better response) instead of switching to $t_{1}$ (which is the best response) and subsequently players 2 and 3 switch to $t_{4}$. This results in the joint strategy $\left(t_{4}, t_{4}, t_{4}\right)$ which is a Nash equilibrium. If $x=t_{4}$ then we have a shorter improvement path $\left(t_{4}, t_{4}, \underline{y}\right) \Rightarrow$ $\left(t_{4}, t_{4}, t_{4}\right)$.

Case 2. $s=\left(t_{2}, x, t_{2}\right)$. If $x \neq t_{3}$ then $\left(t_{2}, \underline{x}, t_{2}\right) \Rightarrow\left(t_{2}, t_{3}, t_{2}\right) \Rightarrow\left(t_{2}, t_{3}, t_{3}\right)$. If $x=$ $t_{3}$ then $\left(t_{2}, x, \underline{t_{2}}\right) \Rightarrow\left(t_{2}, t_{3}, t_{3}\right)$. But the proof of Case 1 shows that $\left(t_{2}, t_{3}, t_{3}\right) \Rightarrow^{*}$ $\left(t_{4}, t_{4}, t_{4}\right)$, so irrespective of the value of $x$ there is a finite improvement path starting in $s$.

In turn, there are social network games which have Nash equilibria but are not weakly acyclic.

Example 32. Consider the modification of the network in Figure 15 where we 'twin' the node with product set $\left\{t_{4}\right\}$, call it 4 , with a new node 5 also with the product set $\left\{t_{4}\right\}$ by adding edges $(4,5)$ and $(5,4)$. Let the weight of each new edge be a constant $w \geq \theta$. From the previous example, it is easy to see that the joint strategy $\left(t_{4}, t_{4}, t_{4}, t_{4}, t_{4}\right)$ (where we list the choice of nodes 1 to 5 ) is a Nash equilibrium. On the other hand, consider the joint strategy $s=\left(t_{1}, t_{1}, t_{2}, t_{0}, t_{0}\right)$. It corresponds to the joint strategy $\left(t_{1}, t_{1}, t_{2}\right)$ analysed in Example 2, where we showed that every improvement path starting at it is infinite. The same holds here and therefore the game is not weakly acyclic.

Theorem 33. For an arbitrary network (respectively, a network whose underlying graph has no source nodes) $\mathcal{S}$, deciding whether the game $\mathcal{G}(\mathcal{S})$ is weakly acyclic is co-NP-hard. 
Proof. Consider the case of an arbitrary network. In the proof of Theorem 28 we showed that if a solution to the instance of the PARTITION problem exists then in the associated game $\mathcal{G}(\mathcal{S})$, there is a joint strategy $s$ such that all improvement paths starting in $s$ are infinite. This implies that $\mathcal{G}(\mathcal{S})$ is not weakly acyclic.

Conversely, if there is no solution to the instance of the PARTITION problem then there is a scheduler $f$ such that all improvement paths in $\mathcal{G}(\mathcal{S})$ that respects $f$ are finite. This implies that $\mathcal{G}(\mathcal{S})$ is weakly acyclic.

The proof for the case of a network whose underlying graph has no source nodes is analogous and uses the proof of Theorem 30 .

\section{Concluding remarks}

\subsection{Summary of the results}

In this paper we studied various aspects of product adoption by agents who form a social network by focussing on a natural class of strategic games associated with the class of social networks introduced in [2]. We identified three natural types of (pure) Nash equilibria in these games: arbitrary, non-trivial, and determined, and focussed on games associated with four classes of social networks: arbitrary ones and those whose underlying graph is a DAG, or a simple cycle, or has no source nodes. We also showed that the price of anarchy and the price of stability is unbounded, even if we limit ourselves to the social networks whose underlying graph is a DAG or has no source nodes.

Further, we studied the finite best response property (FBRP), the finite improvement property (FIP) and also introduced a new class of games that have the uniform FIP. The following table summarizes our complexity and existence results, where we refer to the underlying graph having $n$ nodes.

\begin{tabular}{|l|c|c|c|c|}
\hline \multicolumn{1}{|c|}{ property } & arbitrary & DAG & simple cycle & $\begin{array}{c}\text { no source } \\
\text { nodes }\end{array}$ \\
\hline Arbitrary NE & NP-complete & always exists & always exists & always exists \\
Non-trivial NE & NP-complete & always exists & $O(|\mathcal{P}| \cdot n)$ & $O\left(|\mathcal{P}| \cdot n^{3}\right)$ \\
Determined NE & NP-complete & NP-complete & $O(|\mathcal{P}| \cdot n)$ & NP-complete \\
FBRP & co-NP-hard & yes & $O(|\mathcal{P}| \cdot n)$ & co-NP-hard \\
FIP & co-NP-hard & yes & $?$ & co-NP-hard \\
Uniform FIP & co-NP-hard & yes & yes & co-NP-hard \\
Weakly acyclic & co-NP-hard & yes & yes & co-NP-hard \\
\hline
\end{tabular}

We also mentioned in the Section 11 the notion of a game having the uniform FBRP. In a recent paper, [4], we developed the notion of schedulers further and in particular showed that for finite games the notions of having the uniform FIP and the uniform FBRP are equivalent. This provides another way of showing that the results of the previous section also hold for the latter notion. 


\subsection{Final comments}

In the definition of the social network games we took a number of simplifying assumptions. In particular, we stipulated that the source nodes have a constant payoff $c_{0}>0$. One could allow the source nodes to have arbitrary positive utility for different products. This would not affect the proofs. Indeed, in Nash equilibria the source nodes would select only the products with the highest payoff, so the other products in their product sets could be disregarded. Further, the FBRP, the FIP, the uniform FIP and weak acyclicity of a social network game is obviously not affected by such a modification.

We could also allow the weights to be parametrized by a product. The corresponding expression in the definition of the payoff function would then become $\sum_{j \in \mathcal{N}_{i}^{t}(s)} w_{j i}(t)-\theta(i, t)$. In contrast, as shown in Example 25, such a modification can affect some of the positive results.

Further, the results of this paper can be slightly generalized by using a more general notion of a threshold that would also depend on the set of neighbours who adopted a given product. In this more general setup for $i \in V, t \in P(i)$ and $X \subseteq N(i)$, the threshold function $\theta$ yields a value $\theta(i, t, X) \in(0,1]$.

For the results to continue to hold one needs to assume that the threshold function satisfies the following monotonicity condition: if $X_{1} \subseteq X_{2}$ then $\theta\left(i, t, X_{1}\right) \geq \theta\left(i, t, X_{2}\right)$. Intuitively, agent $i$ 's resistance to adopt a product decreases when the set of its neighbours who adopted it increases. We decided not to use this definition for the sake of readability.

This work can be pursued in a couple of natural directions. One is the study of social networks with other classes of underlying graphs. Another is an investigation of the complexity results for other classes of social networks, in particular for the equitable ones, i.e., networks in which the weight functions are defined as $w_{j i}=\frac{1}{|N(i)|}$ for nodes $i$ and $j \in N(i)$. One could also consider other equilibrium concepts like strict Nash equilibria.

Currently we started a study of slightly different games, in which the players are obliged to choose a product, i.e., games in which the strategy $t_{0}$ is absent. Such games naturally correspond to situations in which the agents always choose a product, for instance a subscription for their mobile telephone. These games substantially differ from the ones considered here. For example, Nash equilibria do not need to exist when the underlying graph is a simple cycle.

Finally, we initiated in [3] an analysis of the consequences of introducing new products in a social network. This can be done using the framework of the social network games by focusing on the finite best response property and the finite improvement property. In particular we found that in some cases such a product addition can lead to a different product selection in which, remarkably, each agent is strictly worse off. 


\section{Acknowledgments}

We would like to thank the referee for an exceptionally detailed and useful report. Theorem 5 was suggested by Bernhard von Stengel.

\section{References}

[1] N. Alon, M. Feldman, A. D. Procaccia, and M. Tennenholtz. A note on competitive diffusion through social networks. Inf. Process. Lett., 110(6):221$225,2010$.

[2] K. R. Apt and E. Markakis. Diffusion in social networks with competing products. In Proc. 4th International Symposium on Algorithmic Game Theory (SAGT11), volume 6982 of Lecture Notes in Computer Science, pages 212-223. Springer, 2011.

[3] K. R. Apt, E. Markakis, and S. Simon. Paradoxes in social networks with multiple products. Manuscript, CWI, Amsterdam, The Netherlands, 2013. Computing Research Repository (CoRR), http://arxiv.org/abs/1301. 7592.

[4] K. R. Apt and S. Simon. A classification of weakly acyclic games. In Proc. 5th International Symposium on Algorithmic Game Theory (SAGT12), volume 7615 of Lecture Notes in Computer Science, pages 1-12. Springer, 2012.

[5] A. Borodin, Y. Filmus, and J. Oren. Threshold models for competitive influence in social networks. In Proc. 6th International Workshop on Internet and Network Economics (WINE 2010), pages 539-550, 2010.

[6] M. Brautbar and M. Kearns. A clustering coefficient network formation game. In Proc. 4th International Symposium on Algorithmic Game Theory (SAGT11), volume 6982 of Lecture Notes in Computer Science, pages 224235. Springer, 2011.

[7] N. Chen. On the approximability of influence in social networks. SIAM J. Discrete Math., 23(3):1400-1415, 2009.

[8] D. Easley and J. Kleinberg. Networks, Crowds, and Markets. Cambridge University Press, 2010.

[9] M. Granovetter. Threshold models of collective behavior. American Journal of Sociology, 83(6):1420-1443, 1978.

[10] J. Howson. Equilibria of polymatrix games. Management Science, 18(5):312-318, 1972.

[11] N. Immorlica, J. M. Kleinberg, M. Mahdian, and T. Wexler. The role of compatibility in the diffusion of technologies through social networks. In J. K. MacKie-Mason, D. C. Parkes, and P. Resnick, editors, ACM Conference on Electronic Commerce, pages 75-83. ACM, 2007. 
[12] E. Janovskaya. Equilibrium points in polymatrix games. Litovskii Matematicheskii Sbornik, 8:381-384, 1968.

[13] M. Kearns, M. Littman, and S. Singh. Graphical models for game theory. In Proceedings of the 17th Conference in Uncertainty in Artificial Intelligence (UAI '01), pages 253-260. Morgan Kaufmann, 2001.

[14] D. Kempe, J. M. Kleinberg, and É. Tardos. Maximizing the spread of influence through a social network. In L. Getoor, T. E. Senator, P. Domingos, and C. Faloutsos, editors, KDD, pages 137-146. ACM, 2003.

[15] I. Milchtaich. Congestion games with player-specific payoff functions. Games and Economic Behaviour, 13:111-124, 1996.

[16] D. Monderer and L. S. Shapley. Potential games. Games and Economic Behaviour, 14:124-143, 1996.

[17] S. Morris. Contagion. The Review of Economic Studies, 67(1):57-78, 2000.

[18] T. Schelling. Micromotives and Macrobehavior. Norton, 1978.

[19] S. Simon and K. R. Apt. Choosing products in social networks. In Proc. 8th International Workshop on Internet and Network Economics (WINE), volume 7695 of Lecture Notes in Computer Science, pages 100-113. Springer, 2012.

[20] É. Tardos and T. Wexler. Network formation games and the potential function method. In N. Nisan, T. Roughgarden, É. Tardos, and V. J. Vazirani, editors, Algorithmic Game Theory, chapter 19, pages 487-516. Cambridge University Press, 2007.

[21] H. P. Young. The evolution of conventions. Econometrica, 61(1):57-84, 1993. 